\title{
mTOR pathway activation drives lung cell senescence and emphysema
}

\author{
Amal Houssaini, ${ }^{1,2}$ Marielle Breau, ${ }^{1}$ Kanny Kebe, 'Shariq Abid, ${ }^{1}$ Elisabeth Marcos, 'Larissa Lipskaia, ${ }^{1}$ \\ Dominique Rideau, ' Aurelien Parpaleix, Jin Huang, 'Valerie Amsellem, ${ }^{1}$ Nora Vienney, ${ }^{1}$ \\ Pierre Validire, ${ }^{3}$ Bernard Maitre, Aya Attwe, Christina Lukas, ${ }^{1}$ David Vindrieux, ${ }^{4}$ Jorge Boczkowski, \\ Genevieve Derumeaux, ${ }^{1}$ Mario Pende, ${ }^{5}$ David Bernard, ${ }^{4}$ Silke Meiners, ${ }^{2}$ and Serge Adnot ${ }^{1}$ \\ INSERM U955, Département de Physiologie-Explorations Fonctionnelles, and DHU A-TVB Hôpital Henri Mondor, AP-HP, \\ Créteil, France. ${ }^{2}$ Comprehensive Pneumology Center (CPC), University Hospital, Ludwig-Maximilians University, Helmholtz \\ Zentrum München, Member of the German Center for Lung Research (DZL), Munich, Germany. Institut Mutualiste \\ Montsouris, Département anatomopathologie, Paris, France. ${ }^{4}$ Centre de Recherche en Cancérologie de Lyon, UMR INSERM \\ U1052/CNRS 5286, Université de Lyon, Centre Léon Bérard, Lyon, France. IINSERM U845, Université Paris Descartes,
} 75015, Paris, France.

Chronic obstructive pulmonary disease (COPD) is a highly prevalent and devastating condition for which no curative treatment is available. Exaggerated lung cell senescence may be a major pathogenic factor. Here, we investigated the potential role for mTOR signaling in lung cell senescence and alterations in COPD using lung tissue and derived cultured cells from patients with COPD and from age- and sex-matched control smokers. Cell senescence in COPD was linked to $\mathrm{mTOR}$ activation, and $\mathrm{mTOR}$ inhibition by low-dose rapamycin prevented cell senescence and inhibited the proinflammatory senescence-associated secretory phenotype. To explore whether mTOR activation was a causal pathogenic factor, we developed transgenic mice exhibiting mTOR overactivity in lung vascular cells or alveolar epithelial cells. In this model, mTOR activation was sufficient to induce lung cell senescence and to mimic COPD lung alterations, with the rapid development of lung emphysema, pulmonary hypertension, and inflammation. These findings support a causal relationship between mTOR activation, lung cell senescence, and lung alterations in COPD, thereby identifying the mTOR pathway as a potentially new therapeutic target in COPD.

Authorship note: MB and KK contributed equally to this work.

Conflict of interest: The authors have declared that no conflict of interest exists.

Submitted: February 2, 2017

Accepted: January 5, 2018

Published: February 8, 2018

Reference information: JCI Insight. 2018;3(3):e93203. https:// doi.org/10.1172/jci.insight. 93203

\section{Introduction}

Chronic obstructive pulmonary disease (COPD) is a major cause of morbidity and mortality in most countries. The dominant risk factor for COPD in industrialized countries is smoking, but aging is also important, along with occupational exposures, respiratory infections early in life, and a history of asthma (1). $\mathrm{COPD}$ is characterized by slowly progressive airflow obstruction and emphysema due to destruction of the lung parenchyma (2). Chronic inflammation is a hallmark of COPD that promotes the development of comorbidities $(3,4)$. The mechanisms leading to lung destruction and inflammation in COPD are incompletely understood, and therefore, no curative treatment is available. One recent concept is that cell senescence plays a key pathophysiological role in COPD (5-7).

Cell senescence is a stable growth arrest that can be triggered by 2 different processes (7-9). Replicative senescence is a consequence of gradual telomere shortening due to incomplete chromosomal replication during somatic cell divisions $(9,10)$. Premature senescence, in contrast, is a response to various stress stimuli, such as oxidative stress or oncogenic insults (9). Senescent cells exhibit a complex phenotype characterized by permanent cell cycle arrest mediated by the $\mathrm{p} 53$ and/or $\mathrm{p} 16 / \mathrm{Rb}$ pathways; increased cell size; altered cell morphology; resistance to apoptosis; altered gene expression, including $\beta$-galactosidase ( $\beta$-Gal) upregulation; and a unique secretory phenotype known as the senescence-associated secretory phenotype (SASP) (8). We previously reported that a characteristic feature of COPD is the accumulation of senescent cells in the lung, which limits the potential for tissue renewal and contributes to chronic inflammation through the SASP (11-13). Whether cell senescence plays a causal role in the pathogenesis of COPD, however, remains unknown, and the mechanisms underlying cell senescence in COPD need to be clarified. A crucial point is whether a specific mechanism is shared by several cell types in the lung and may constitute a target for pharmacological treatments. 
The mTOR signaling pathway is well known to be involved in cell metabolism, survival, and growth (14). Several lines of evidence also indicate that mTOR is a major cell senescence pathway (15). Activation of mTOR induced by deletion of phosphatase and tensin homolog PTEN or expression of constitutively active serine/threonine kinase Akt induces senescence of normal mouse or human cells $(16,17)$. Rapamycin inhibits mTOR activity and is one of the few drugs capable of prolonging life in many species (18). Within the cell, mTOR is found in 2 distinct protein complexes, mTORC1 and mTORC2 (19-21), which differ regarding their upstream activators, downstream effectors, and sensitivity to inhibition by rapamycin. The mTORC1 substrates include S6 kinases (S6K) and 4E-BP, whereas mTORC2 phosphorylates the hydrophobic motif of Akt family members at $\mathrm{Ser}^{473}$, leading to subsequent phosphorylation of downstream effectors such as GSK3. Short-term rapamycin exposure blocks mTORC1 but not mTORC2, in contrast to long-term exposure, which inhibits both complexes. Rapamycin treatment can affect the cell senescence process and SASP, but it does so with differential effects depending on stress or cell type $(7,17,22)$.

Here, we explored the potential role for the mTOR signaling pathway in lung cell senescence in COPD by studying lung specimens and cultured cells derived from patients with COPD and from age- and sexmatched control smokers. Cell senescence was linked to mTOR activation, and mTOR inhibition by lowdose rapamycin prevented cell senescence and inhibited the SASP. To determine whether mTOR activation had a causal role in lung pathology, we developed transgenic mice exhibiting mTOR overactivity in several lung cell types. In this model, mTOR activation was sufficient to induce cell senescence in the lung and to mimic the lung alterations seen in COPD, including the rapid development of lung emphysema, pulmonary hypertension, and inflammation.

\section{Results}

The mTOR signaling pathway is activated in lungs and cultured cells derived from patients with COPD. To investigate whether cell senescence and mTOR activity are linked in COPD, we investigated lung tissues from 30 patients with COPD and 30 controls, as well as cultures derived from lung cells obtained from a subset of 13 patients and 16 controls. Supplemental Tables 1 and 2 (supplemental material available online with this article; https:// doi.org/10.1172/jci.insight.93203DS1) report the features of patients and controls, respectively. In lungs from patients with COPD compared with controls, we found markedly higher protein levels of Ser473 phosphorylated Akt (p-Akt ${ }^{\mathrm{Ser} 43}$ ) and of phosphorylated GSK3, S6K, and 4E-BP1 (Figure 1A), together with higher protein levels of p16 and p21. Lung expression of p16 correlated positively with p-Akt (Figure 1A) and with p-GSK3, p-S6K, and p-4E-BP1 proteins levels (Supplemental Figure 1). Immunofluorescence and colocalization studies showed that p16 staining localized predominantly to pulmonary vascular endothelial cells (P-ECs), pulmonary-artery smooth muscle cells (PA-SMCs), and alveolar epithelial cells (AECs) in lungs from patients with COPD (Figure 1B). In contrast to p-Akt ${ }^{\mathrm{Ser} 473}$ levels, $\mathrm{p}$-Akt ${ }^{\mathrm{Thr} 308}$ levels were lower in lungs from patients with COPD than in those from controls (Supplemental Figure 1).

Cultured PA-SMCs (Figure 2A) or P-ECs (Figure 2B) were studied after serum deprivation for 48 hours or 24 hours, respectively. Cells from patients with COPD were characterized by a higher number of senescent cells, as assessed by the percentage of $\beta$-Gal-positive cells; loss of proliferative capacity as determined by Ki67 staining; and higher levels of p-Akt ${ }^{\text {Ser473 }}$, p-GSK3, p-S6K, and p-4E-BP1 proteins compared with those from controls. Senescent PA-SMCs from patients with COPD that stained positive for $\mathrm{p} 16$ showed strong staining for $\mathrm{p}-\mathrm{Akt}^{\mathrm{Ser} 473}$ (Figure 2A), whereas those from controls stained neither for $\mathrm{p} 16$ nor for $\mathrm{p}-\mathrm{Akt} \mathrm{ter}^{\mathrm{S} 473}$.

Inhibition of $m$ TOR signaling by rapamycin delays the onset of replicative cell senescence in COPD. To question whether the onset of cellular senescence is causally related to the observed activation of the mTOR pathway, we investigated whether mTOR inhibition affected cell senescence in COPD. To this end, we compared cultured PA-SMCs and P-ECs obtained from patients with COPD and controls after treatment with rapamycin or vehicle at cell isolation and at each culture passage. A rapamycin dose of $10 \mathrm{nM}$, which did not affect the cell-growth rate, was applied, and the cell population doubling level (PDL) was determined. The PDL was lower for PA-SMCs (Figure 3A) and P-ECs (Figure 3B) from patients with COPD compared with those from controls. Rapamycin treatment consistently increased the PDLs of both PA-SMCs and P-ECs from patients with COPD and, to a lesser extent, from controls. Thus, PDLs for PA-SMCs and P-ECs from patients with COPD and controls no longer differed when both were treated with rapamycin (Figure 3, A and B). Rapamycin treatment also decreased the number of $\beta$-Gal-positive cells to similar values in the COPD and control groups (Figure 3, A and B). 
A

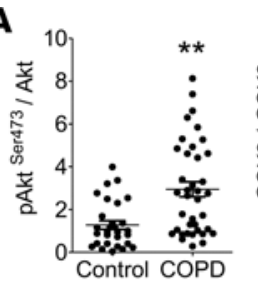

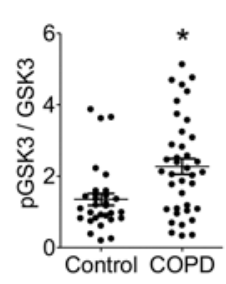

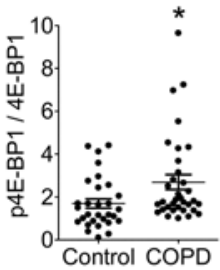

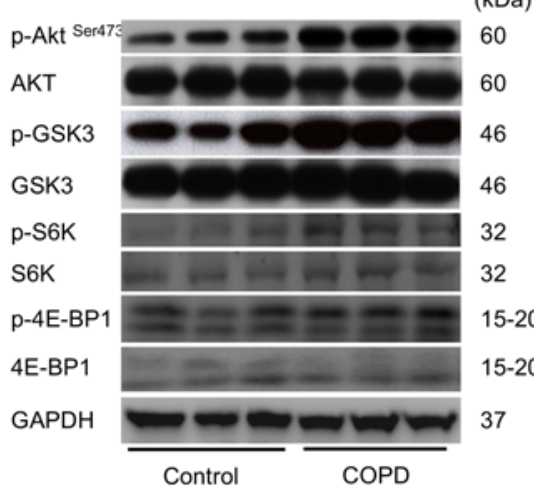

Smooth muscle cells
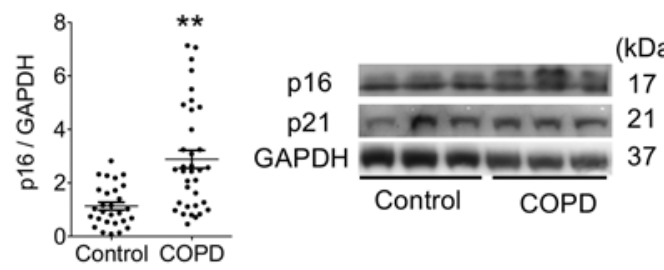

Control $\frac{}{\text { COPD }}$

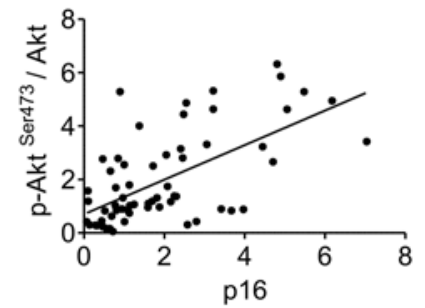

Alveolar epithelial cells

Control

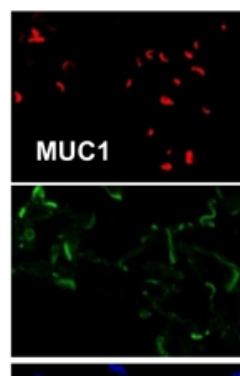

COPD
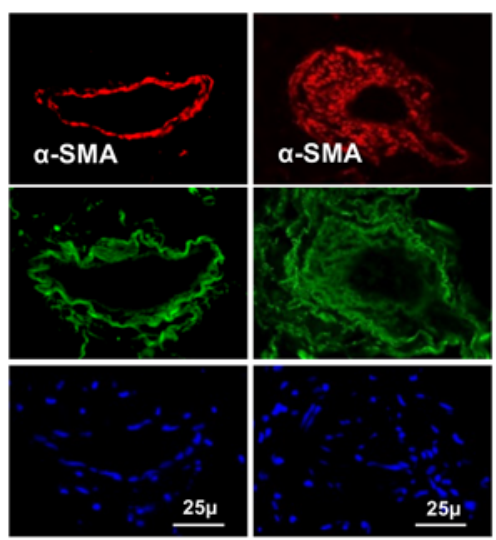

$25 \mu$

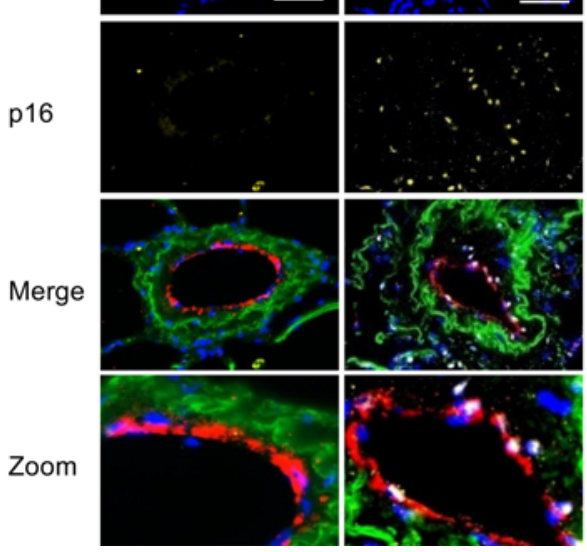

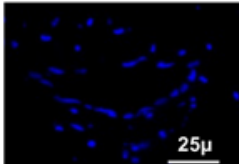
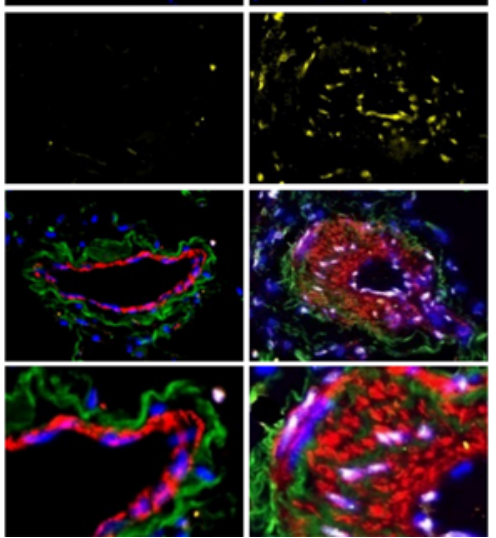
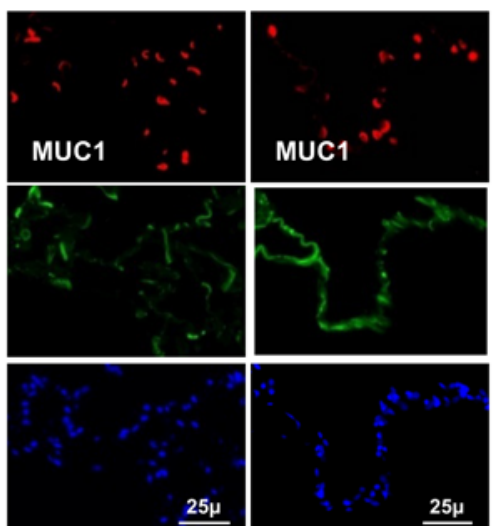

$25 \mu$
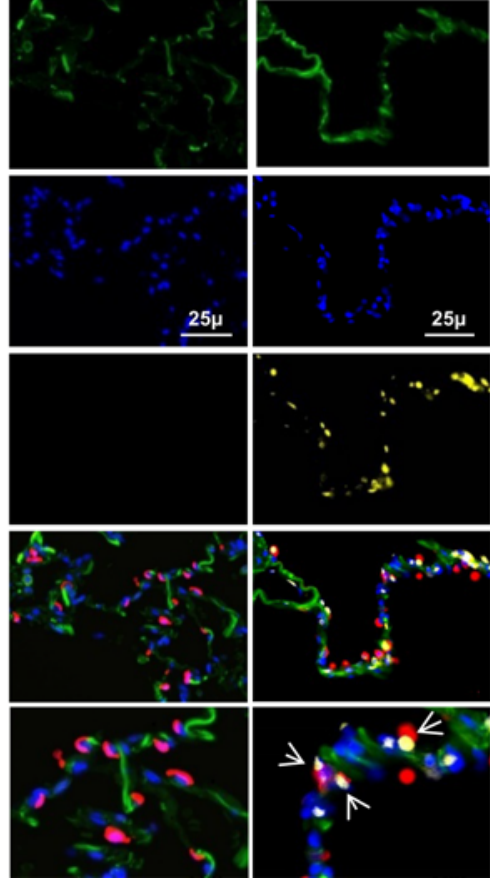

Figure 1. Analysis of lung samples from 30 patients with COPD and $\mathbf{3 0}$ controls. (A) Protein levels of Akt phosphorylated at Ser473 (Akt-Ser473), glycogen synthase kinase 3 (CSK3), S6 kinase (S6K), and 4E-binding protein 1 (4E-BP1), and of p16 and p21 proteins, measured in the lungs relative to GAPDH, using Western blot, in patients with COPD and controls. Values are mean $\pm \mathrm{SEM},{ }^{*} P<0.05$, ${ }^{* *} P<0.01$ compared with values from controls. Positive correlation between $\mathrm{p}$-Akt-Ser473 and p16 protein levels (Spearman $\mathrm{r}=0.59 ; P<0.001$, by 2-tailed unpaired $t$ test) in patients with COPD and controls. (B) From left to right, representative photographs of von Willebrand factor-positive endothelial cells, $\alpha$-SMA-positive smooth muscle cells, and $\alpha$-MUC1-positive alveolar epithelial cells also stained for p16. Scale bars: $25 \mu \mathrm{m}$.

Changes in mTOR activity also differed between cells from patients with COPD and controls. In cells from controls whose mTOR activity was lower compared with cells from patients with COPD, both the mTORC2 substrates $\mathrm{p}-\mathrm{Akt}^{\mathrm{Ser} 43}$ and $\mathrm{p}-\mathrm{GSK} 3$ and the mTORC1 substrates $\mathrm{p}$-S6K and $\mathrm{p}-4 \mathrm{E}-\mathrm{BP} 1$ usually increased during successive cell passages, in accordance with the increase in the $\beta$-Gal-positive cell count (Figure 3C and Supplemental Figure 2). Such changes in mTOR effectors were less marked or absent in cells from patients with COPD, which already had a high $\beta-G a l-$ positive cell count and high mTOR activity at 


\section{A Smooth muscle cells}
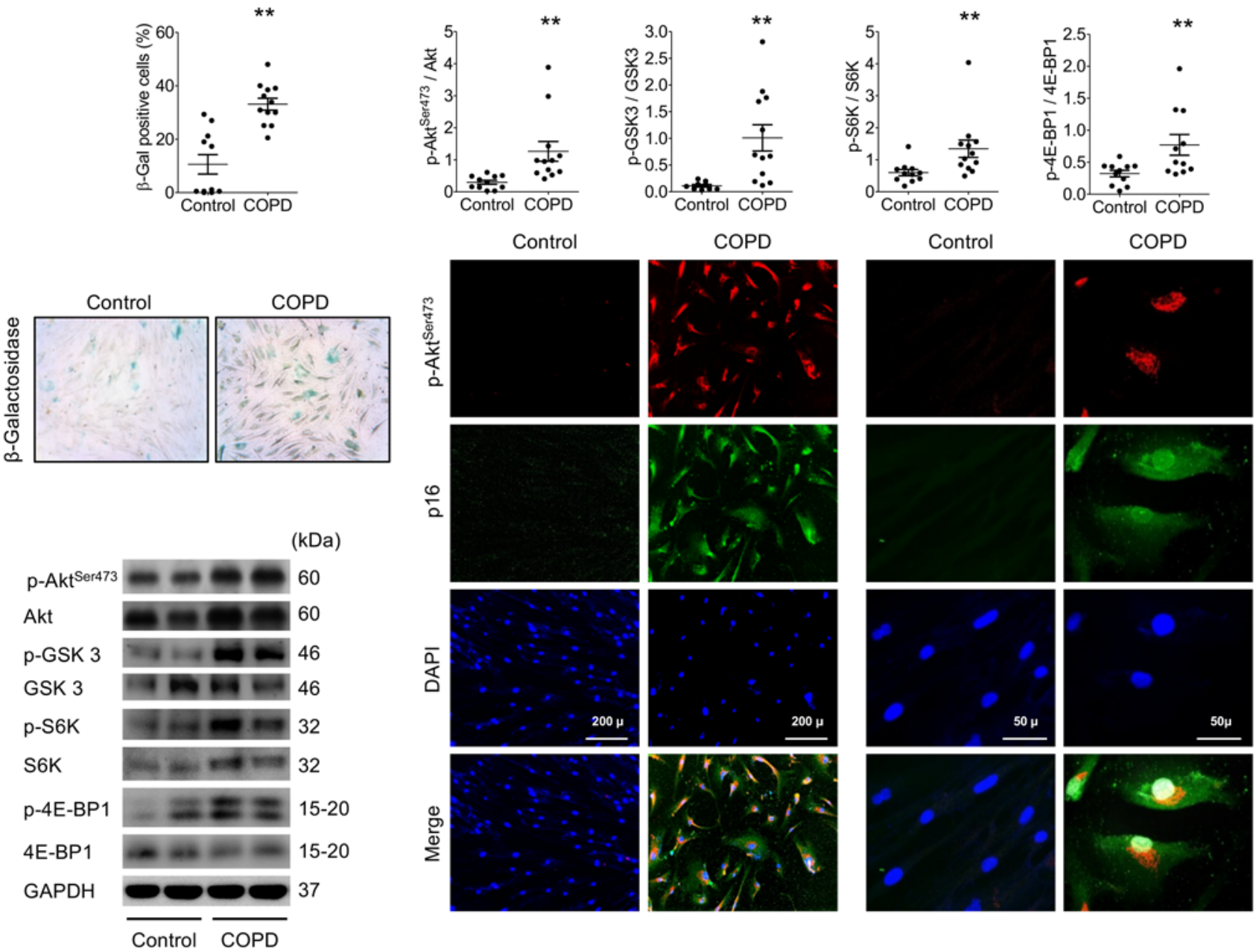

\section{B Endothelial cells}
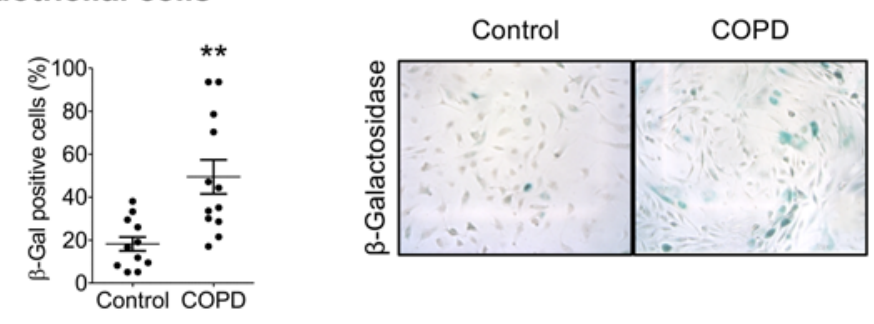

$(\mathrm{kDa})$
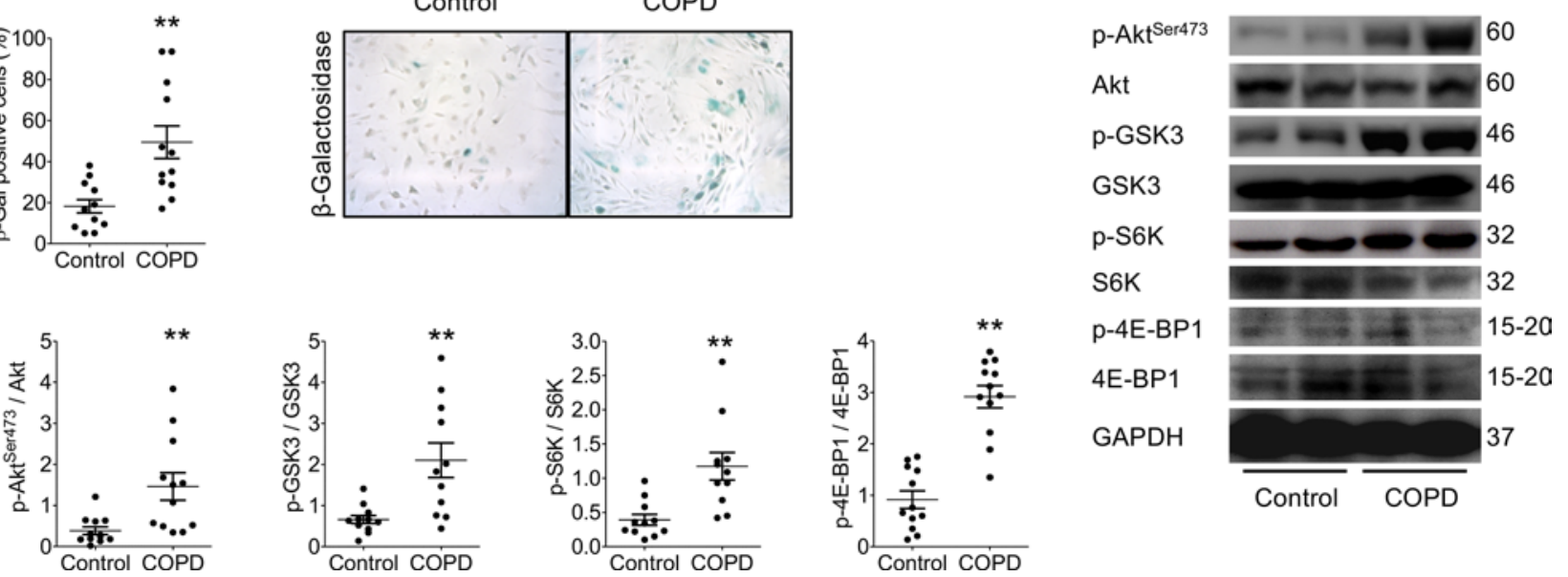

Figure 2. Analysis of cultured cells from patients with COPD and controls. (A) Percentage of $\beta$-Gal-positive cells and protein levels of Akt phosphorylated at Ser473 (Akt-Ser473), glycogen synthase kinase 3 (CSK3), S6 kinase (S6K), and 4E-binding protein 1 (4E-BP1) proteins in cultured pulmonary artery smooth muscle cells (PA-SMCs) from 11 controls and 12 patients with COPD at the earliest cell passage. Values are mean \pm SEM. ${ }^{* *} P<0.01$ vs. controls, by 2 -tailed $t$ test. Representative photographs of PA-SMCs from patients with COPD and controls stained for senescence-associated $\beta$-Cal activity at the corresponding cell passage (left panel; original magnification, 10×). Representative photographs of PA-SMCs from patients with COPD and controls costained for p-Akt and p16 (right panels), illustrated with their respective gels. Scale bars: middle panel $200 \mu \mathrm{m}$ and right panel $25 \mu \mathrm{m}$. (B) Similar representations for pulmonary vascular endothelial cells (P-ECs) from 12 patients with COPD and 12 controls. These measurements were performed in cells deprived of serum for 24 hours (P-ECs) or for 48 hours (PA-SMCs). 


\section{A Smooth muscle cells}

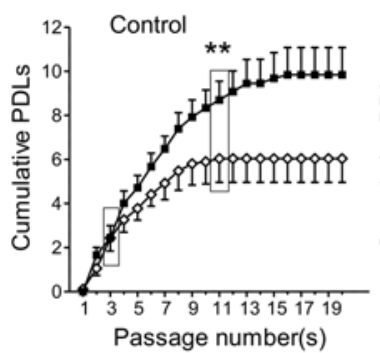

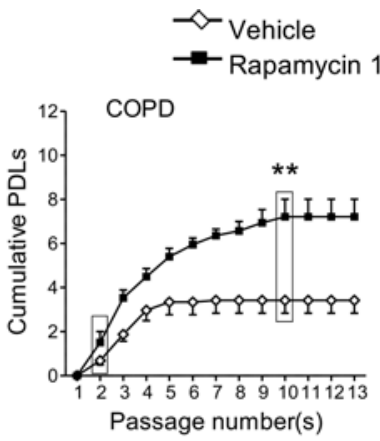

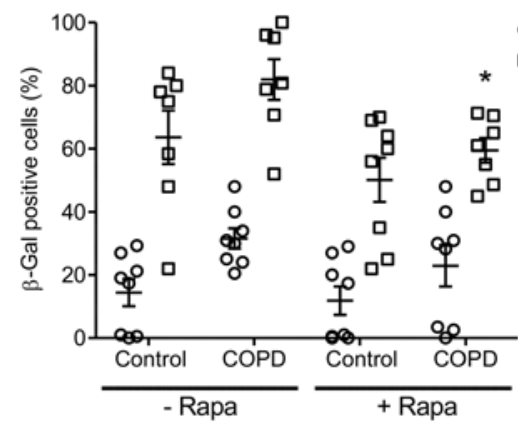

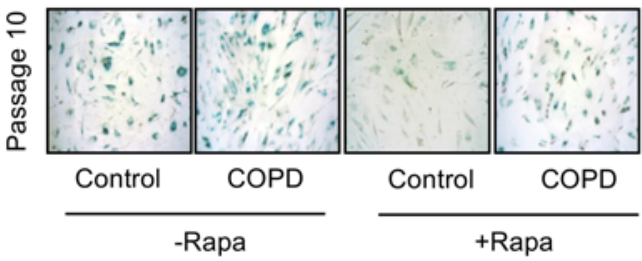

- Passage 2

ㅁ Passage 10

\section{B Endothelial cells}

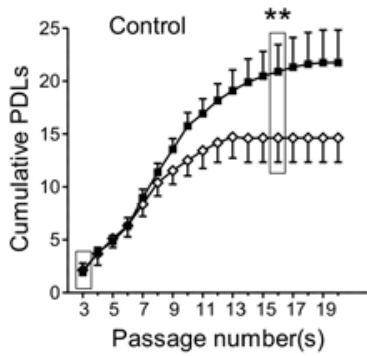

$\sim$ Vehicle

$\rightarrow$ Rapamycin 10nM

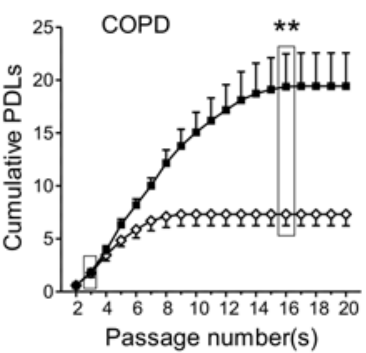

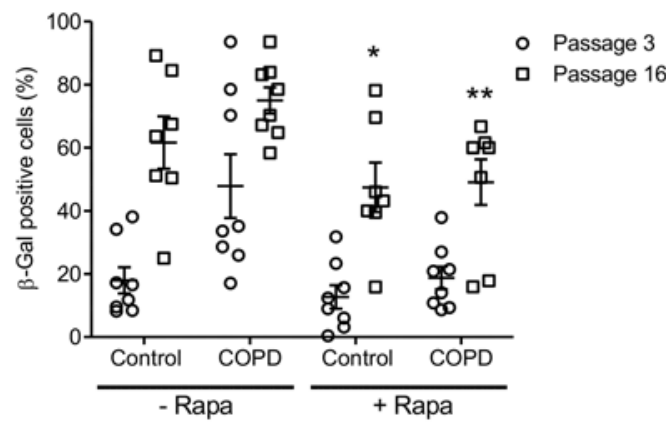

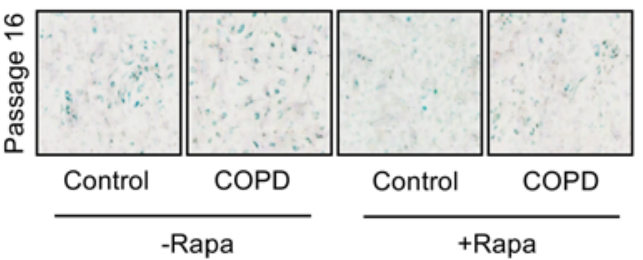

\title{
C Smooth muscle cells
}
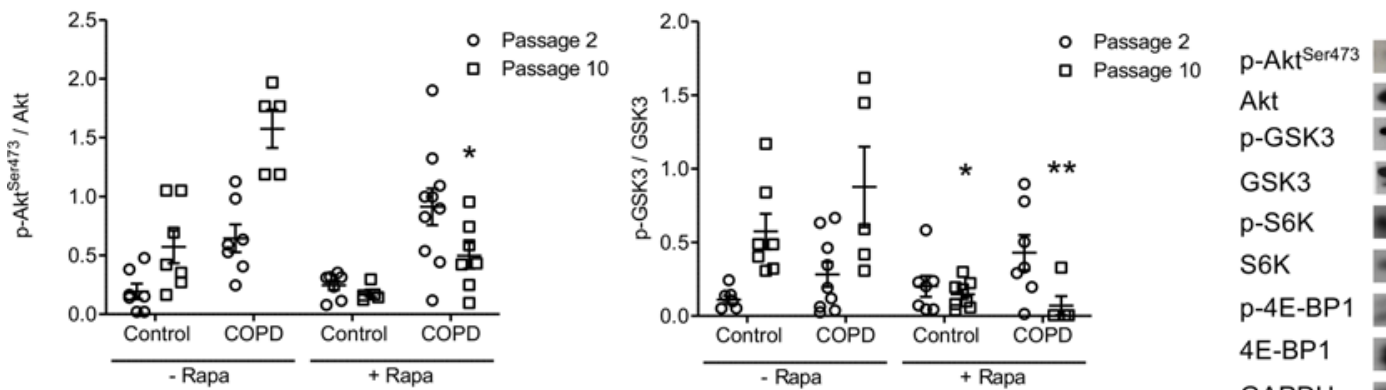

(kDa)

- Rapa + Rapa

- Rapa

p-S6K

S6K
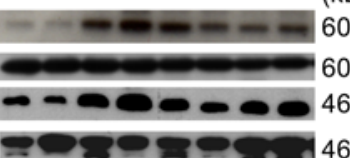

p-4E-BP1
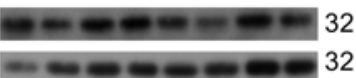

4E-BP1

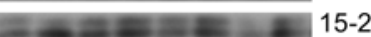

GAPDH
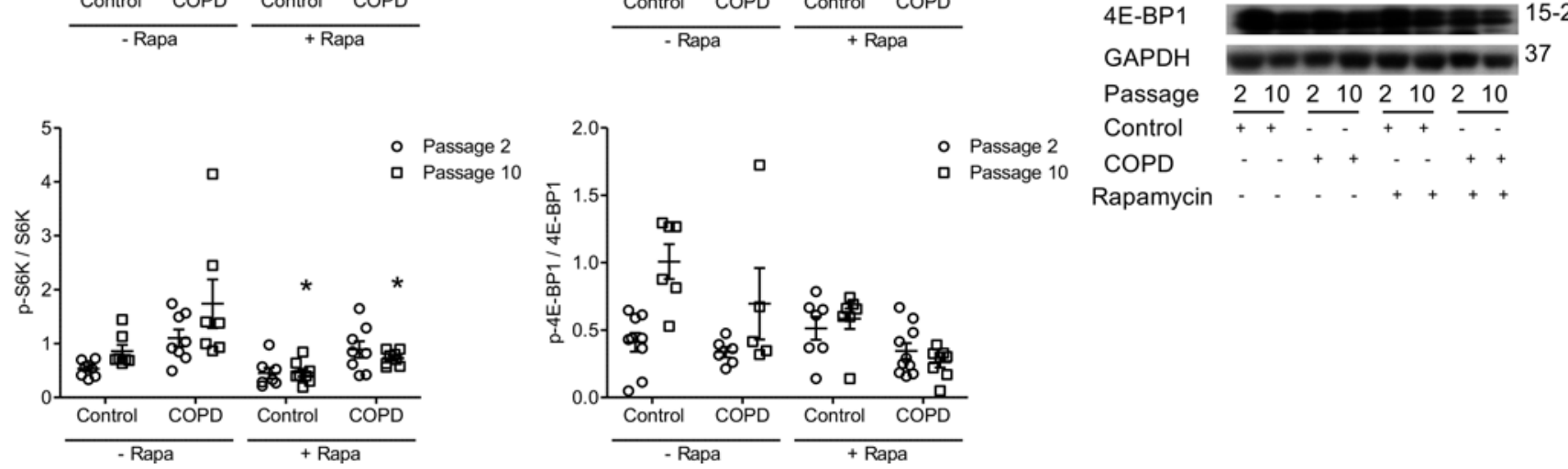

Passage $\quad 2 \quad 10 \quad 2 \quad 10 \quad 2 \quad 10 \quad 2 \quad 10$

Control

COPD

Rapamycin

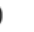

(1)

(n)

$5-20$ 
phosphorylated at Ser473 (Akt-Ser473), glycogen synthase kinase 3 (GSK3), S6 kinase (S6K), and 4E-binding protein 1 (4E-BP1) in PA-SMCs from controls and patients with COPD at passages 2 and 10 , during vehicle or rapamycin treatment. Values are mean \pm SEM. ${ }^{*} P<0.05$, ${ }^{* *} P<0.01$ compared with vehicle-treated cells at the corresponding cell passage (2-way ANOVA with Bonferroni posthoc test). These measurements were performed in cells deprived of serum for 24 hours (P-ECs) or for 48 hours (PA-SMCs).

early cell passages. Rapamycin consistently reduced the levels of these mTOR effectors in PA-SMCs and $\mathrm{P}-\mathrm{EC}$ and from patients with COPD at senescence, and less consistently in cells from controls (Figure 3C and Supplemental Figure 2). Because one of the rapamycin effects was to inhibit the mTORC1 substrate S6K, we evaluated whether selective S6K1 inhibition mimicked the effects of rapamycin. As shown in Supplemental Figure 3, exposure of P-ECs from patients with COPD to the S6K1 inhibitor (PF478671) in various doses did not inhibit cell senescence, in contrast to rapamycin exposure. In additional experiments, we examined the effects of the potent antioxidant N-acetyl cysteine (NAC). As shown in Supplemental Figure 4, NAC in various doses failed to protect cells from patients with COPD or controls against cell senescence.

Rapamycin inhibits cell senescence in COPD, independently of its effect on the SASP. To determine whether rapamycin affected the SASP of senescent cells from patients with COPD, we measured the secretion of the proinflammatory cytokines IL-6, IL-8, and CCL2 by PA-SMCs and P-ECs from patients with COPD and controls. As shown in Figure 4, A and B, the levels of IL-6, IL-8, and CCL2 measured by ELISA were usually higher in media of PA-SMCs or P-ECs from patients with COPD than in those from controls at similar cell passages. Rapamycin (10 nM) reduced IL-6, IL-8, and CCL2 levels in cells derived from COPD patients and from controls, although the effects varied among patients. We also evaluated whether rapamycin treatment affected cell senescence or the SASP in a curative manner. Rapamycin was added to P-EC or PA-SMC cultures containing over $50 \%$ of $\beta$-Gal-positive cells. As shown in Supplemental Figure 5, rapamycin exposure for 48 hours in doses ranging from 5-20 nM significantly reduced cytokine expression but not $\beta$-Gal-positive cell counts. To further investigate whether suppressing the release of SASP components contributed to the protective effect of rapamycin against cell senescence, we examined the consequences of inhibiting NF- $\mathrm{KB}$, a key transcriptional activator of the inflammatory SASP components. To this end, we transduced the gene encoding the I $\kappa \mathrm{B} \alpha$ super-repressor ( $\mathrm{I} \kappa \mathrm{B} \alpha \mathrm{M})$, a stabilized NF- $\kappa \mathrm{B}$ inhibitor (23), in PA-SMCs. I $\mathrm{B} \alpha \mathrm{M}$ expression in PA-SMCs reduced the expression of IL-6, IL-8, and CCL 2 compared with cells infected with a control retroviral vector, but it had no effect on PDL and the percentage of $\beta$-Gal-positive cells (Figure 4C).

Activation of mTOR signaling by TSC1 deletion is sufficient to induce cell senescence in targeted cells in mice, resulting in COPD-like lung alterations. To assess whether overactivation of $\mathrm{mTOR}$ triggers induction of senescence in targeted cells of the lungs and thereby contributes to the development of COPD, we generated mice with constitutive or conditional deletion of the tuberous sclerosis complex heterodimer TSC1 (a negative mTORC1 regulator) in SMCs (SM22-TSC1 ${ }^{-/-}$mice) (24), ECs (PDGF-TSC1 ${ }^{-/-}$mice) (25), or AECs (SPC-TSC1 ${ }^{-/}$mice) (26). Constitutive SM22-TSC1 ${ }^{-/-}$mice spontaneously developed pulmonary hypertension as previously described (24). Their lungs contained p16-positive cells confined to SMCs in the media of pulmonary vessels (Figure 5A). Cultured PA-SMCs from SM22-TSC1 ${ }^{-1}$ mice exhibited early-onset replicative cell senescence compared with those from control mice, with a lower PDL (Figure 5B) and a higher percentage of $\beta$-Gal-positive cells (Figure 5C). The levels of p-Akt ${ }^{\mathrm{Ser}-473}$, p-GSK-3, p-S6K, and p-4E-BP1 were higher in PA-SMCs from SM22-TSC1 ${ }^{-/-}$mice than in those from control mice, and they even increased during replicative cell senescence (Figure 5D). Rapamycin treatment increased the PDL in PA-SMCs from SM22-TSC1 ${ }^{-/-}$mice, decreased the $\beta$-Gal-positive cell count, and inhibited mTOR activity (Figure 5, B-D). In contrast, rapamycin treatment did not affect the PDL of cells from control mice (Figure 5B).

Mice with conditional TSC1 deletion in ECs (PDGF-TSC1 ${ }^{-/-}$mice) or AECs (SPC-TSC1 ${ }^{-/}$mice) were studied 3 and 6 months after pharmacological Cre recombinase activation. In both transgenic mice strains, TSC1 deletion in targeted cells (Figure 6A and Figure 7A) was associated with higher lung levels of p-Akt ${ }^{\mathrm{Ser}-473}$, p-GSK-3, p-S6K, and p-4E-BP1 after 3 months and a further increase after 6 months, together with a decrease in p-Akt ${ }^{\text {thr308 }}$ (Figure 6B, Figure 7B, and Supplemental Figure 6, A and B). These changes were associated with a time-dependent increase in the lung levels of p16 and p21 proteins following TSC1 deletion (Figures 6B and Figure 7B) and with higher 6-month counts of p16-stained cells among pulmonary vessel cells and AECs (Supplemental Figure 7). Only a few TUNEL-positive cells were seen at baseline, with no changes induced by TSC1 deletion (Supplemental Figure 8, A and B). Compared with controls, both PDGF-TSC1 ${ }^{-/}$and SPC-TSC1 ${ }^{-1}$ 


\section{A Smooth muscle cells}
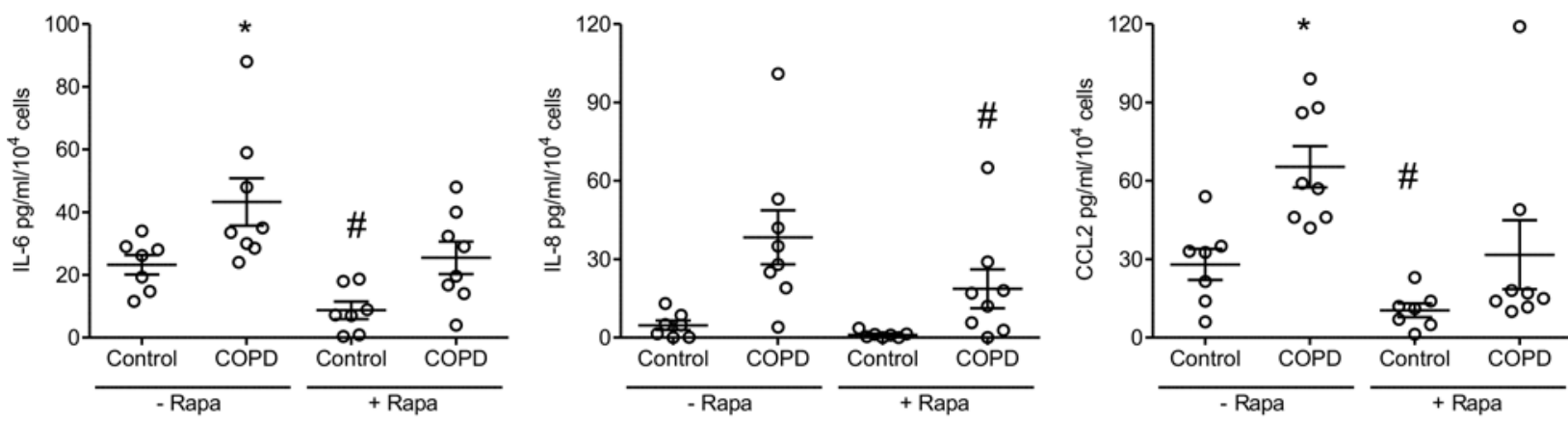

\section{B Endothelial cells}
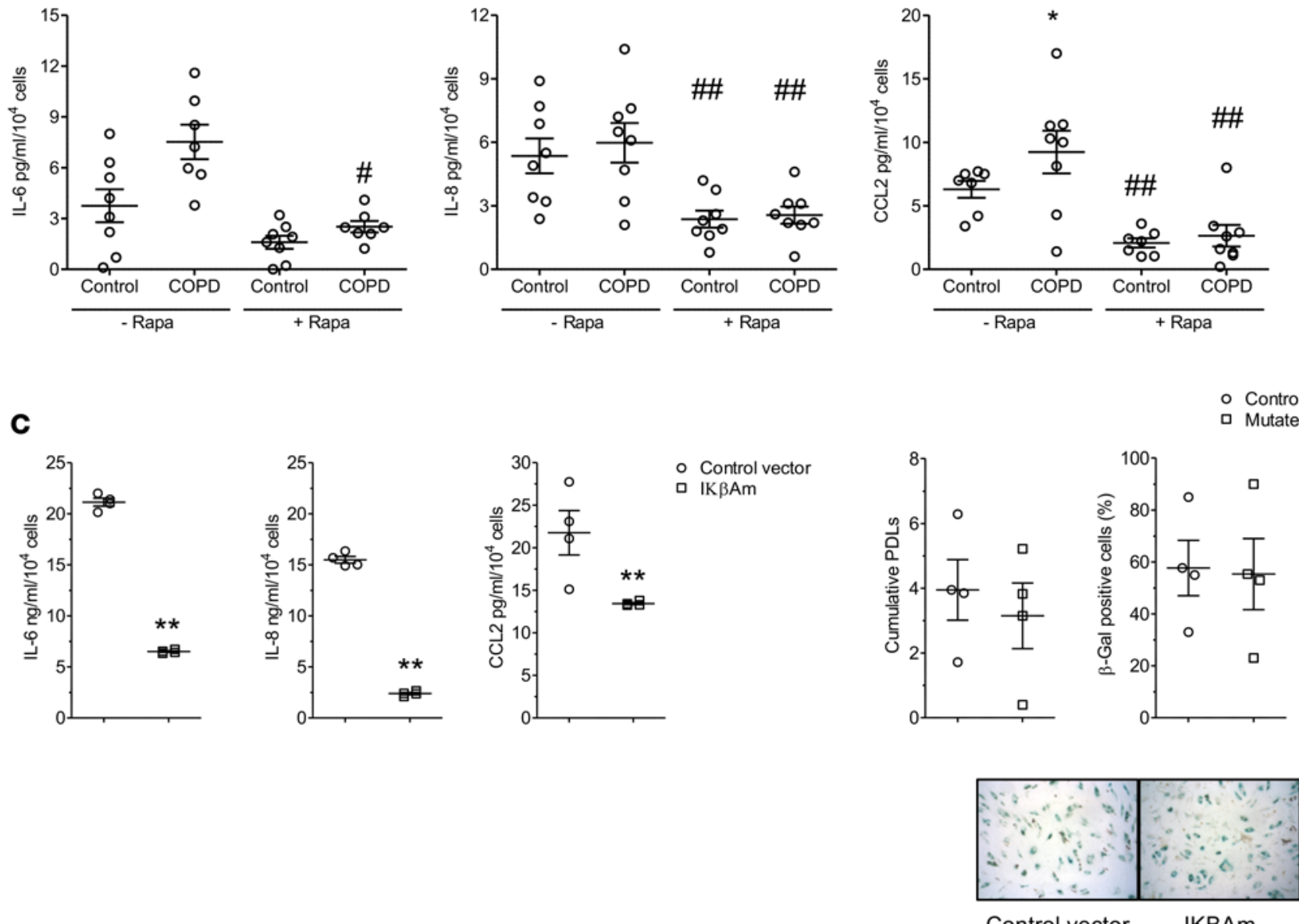

Figure 4. Effect of rapamycin treatment on synthesis of pro-inflammatory cytokines. Effect of rapamycin treatment on the levels of proinflammatory cytokines (IL-6, IL-8, and CCL2) measured in conditioned media of PA-SMCs (A) from 7 patients with COPD and 8 controls, and P-ECs (B) from 8 patients with COPD and 8 controls. Values are mean \pm SEM. ${ }^{*} P<0.05$, compared with values from controls, ${ }^{*} P<0.05$, ${ }^{\#} P<0.01$ compared with vehicle-treated cells, by 2-way ANOVA. (C) Levels of cytokines released by PA-SMCs treated with a retroviral vector expressing the NF- $\kappa B$ inhibitor I $1 \mathrm{~B} \alpha \mathrm{M}$, compared with the levels released by cells treated with the control vector. The graphs show levels of IL-6, IL-8, and CCL2 in PA-SMC-conditioned media, cumulative PDL, and percentage of $\beta$-Gal-positive cells. ${ }^{* *} P<0.01$ compared with values in cells infected with the control vector, by 2 -tailed unpaired $t$ test. These measurements were performed in cells deprived of serum for 24 hours (P-ECs) or for 48 hours (PA-SMCs). 
A
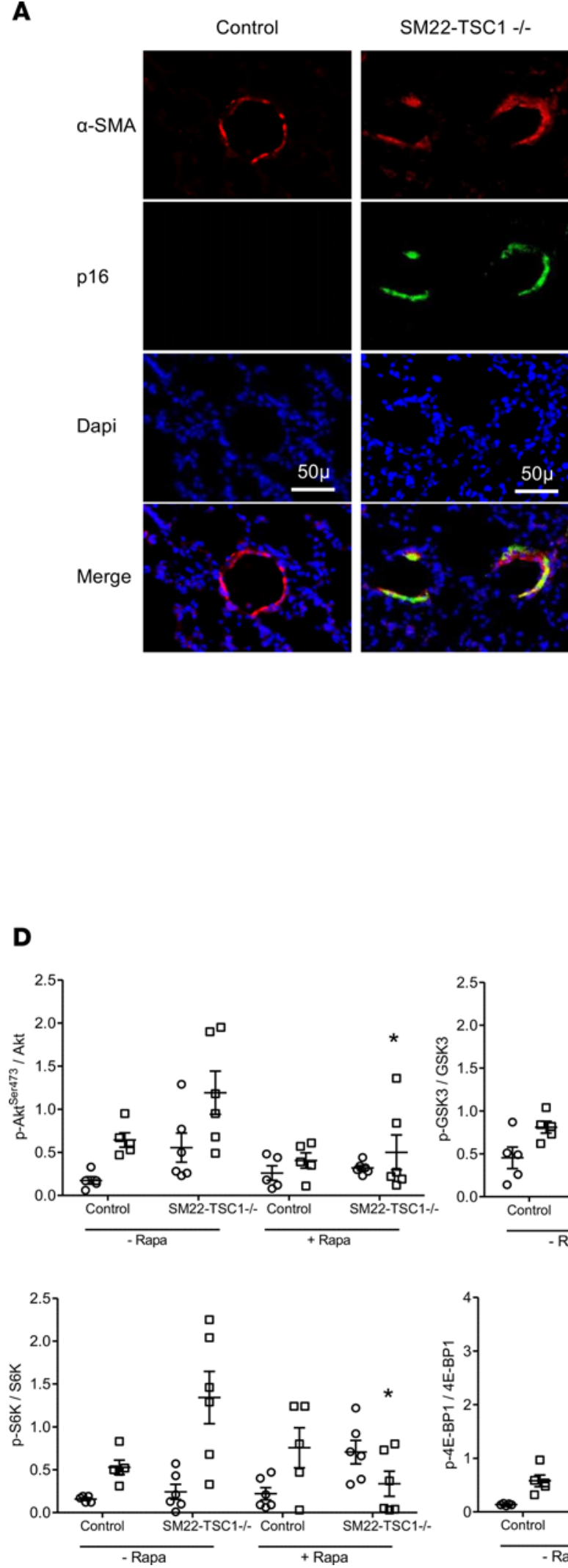

B
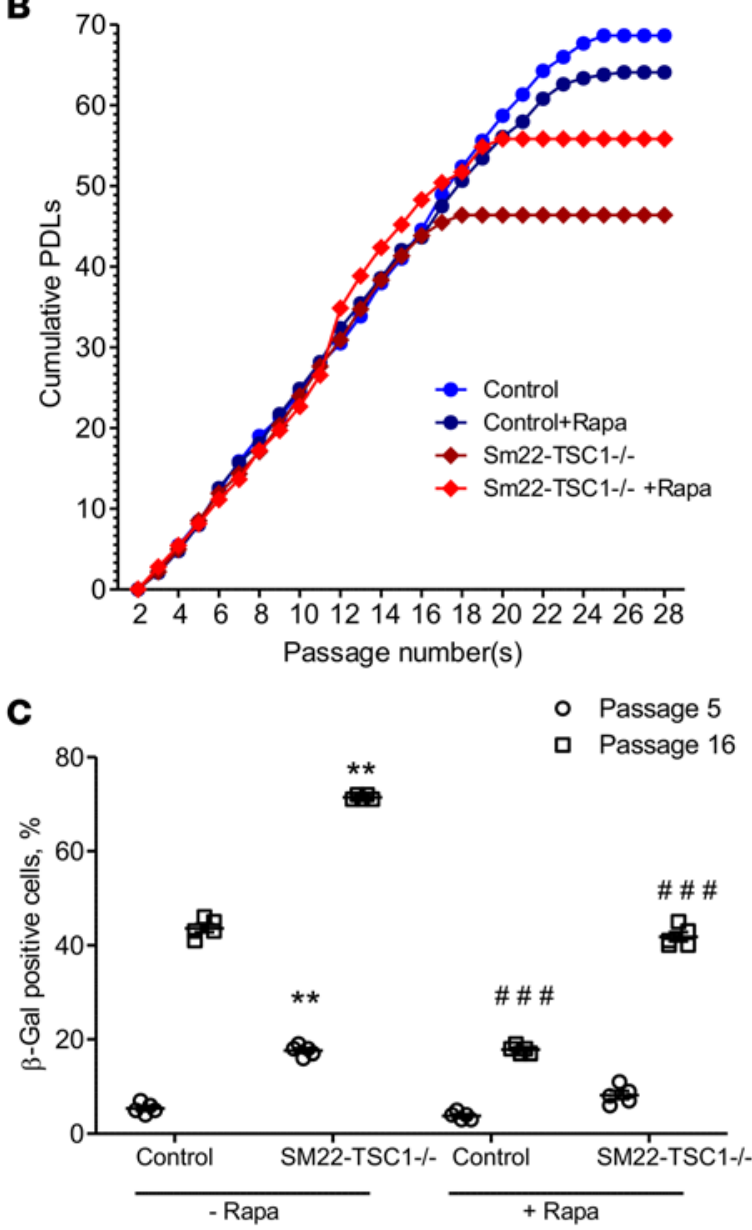

- Passage 5

口 Passage 16

(kDa)

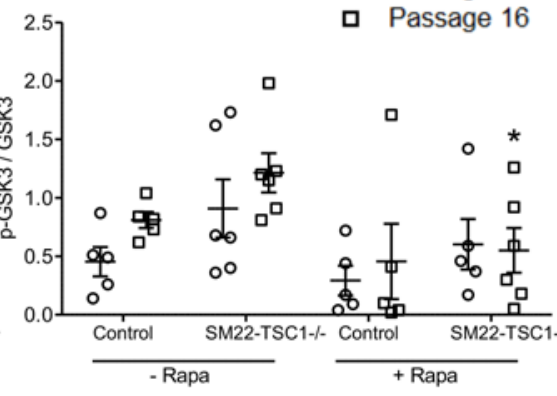

p-Akt ${ }^{\text {Ser473 }}$

Akt

p-GSK3

GSK3

p-S6K

S6K

p-4E-BP1

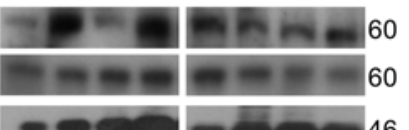

4E-BP1

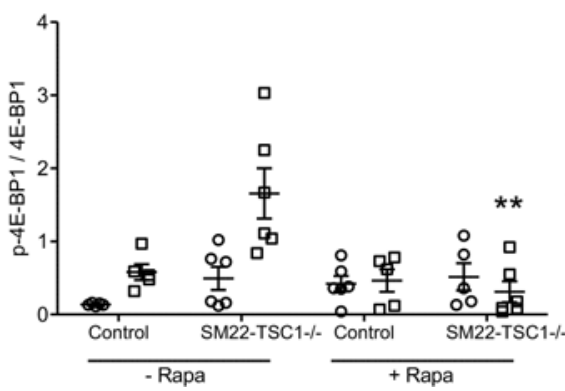

GAPDH

Passage

Control

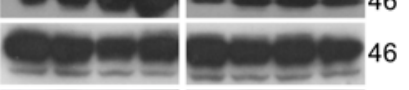

$-0-6-0-32$

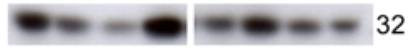

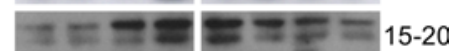

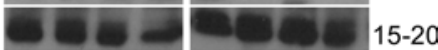

SM22-TSC1-/-

Rapamycin

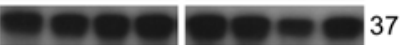

$\begin{array}{llllllll}5 & 16 & 5 & 16 & 5 & 16 & 5 & 16\end{array}$

Figure 5. Characteristics of PA-SMCs from SM22-TSC1-/- mice. (A) Photomicrographs of pulmonary vessel walls showing p16-positive cells also stained for $\alpha$-smooth muscle actin ( $\alpha$-SMA) in SM22-TSC1 ${ }^{-1-}$ mice compared with control mice. Scale bars: $50 \mu \mathrm{m}$. (B) Replicative senescence of PA-SMCs from SM22$\mathrm{TSC1}^{-1-}$ and control mice treated by rapamycin or vehicle $(n=5-6$ in each group). Cells were subjected to repeated passages and counted at each passage, and the population doubling level (PDL) was calculated. (C) Percentage of $\beta$-galactosidase-positive ( $\beta$-Gal-positive) PA-SMCs from SM22-TSC1-1- and control mice at passages 5 and 16 . Data are mean \pm SEM. ${ }^{* *} P<0.01$ vs. controls at the corresponding cell passage, ${ }^{\# \# ~} P<0.001$ compared with vehicle-treated 
cells at the corresponding cell passage, by 2-way ANOVA. (D) Representative Western blots and graphs of protein levels of Akt phosphorylated at Ser473 (Akt-Ser473), glycogen synthase kinase 3 (CSK3), S6 kinase (S6K), and 4E-binding protein 1 (4E-BP1) in PA-SMCs from SM22-TSC1 ${ }^{\prime-}$ and control mice at passages 5 and 16, during treatment with either vehicle or rapamycin. Values are mean \pm SEM. ${ }^{*} P<0.05$, ${ }^{* *} P<0.01$ compared with vehicle-treated cells at the corresponding cell passage (analysis by 2-way ANOVA with Bonferroni posthoc test).

mice developed lung emphysema, as quantified by the mean distance separating alveolar septa (mean linear intercept) and alveolar density (Figure 8A). $\mathrm{PDGF}^{-\mathrm{TSC}^{-/}}{ }^{-}$and SPC-TSC1 ${ }^{-1}$ mice also developed pulmonary hypertension and pulmonary vascular remodeling, as shown by quantification of right ventricular systolic pressure (RVSP), right heart hypertrophy, and increased degree of vascular muscularization (Figure 8B). These alterations were associated with increased lung cytokine levels and lung infiltration by macrophages, as shown by numerous CD68-positive cells in lungs from PDGF-TSC1 ${ }^{-/}$and $\mathrm{SPC}-\mathrm{TSC}^{-/-}$mice studied after induction of TSC1 deletion, which appeared to be distributed homogeneously in the lung parenchyma except for a greater density in perivascular areas (Supplemental Figure 9, A and B). Most importantly, treatment with rapamycin for up to 3 months in PDGF-TSC1 ${ }^{-/}$prevented the development of lung emphysema and pulmonary hypertension, as shown by both structural and functional parameters (Figure 9, A-D). The increased mTOR signaling and higher cytokine levels measured 3 months after tamoxifen exposure in these mice returned to normal after rapamycin treatment (Figure 10, A and B).

In accordance with the fact that mTOR is an essential negative regulator of autophagy, we found that the autophagy proteins LC3II/I, ATG3, and ATG5 were decreased in lungs from PDGF-TSC1 ${ }^{-/}$and SPC-TSC1 ${ }^{-/}$mice (Supplemental Figure 6, A and B). The levels of lung Sirtuin 1 (SIRT1), a stimulator of autophagy, were also decreased in lungs from PDGF-TSC1 ${ }^{-/}$and SPC-TSC1 $1^{-1-}$ mice as previously shown in mice developing emphysema due to cigarette smoke exposure (Supplemental Figure 6, A and B). In contrast, lung emphysema developed in PDGF-TSC1 $1^{-/-}$and SPC- $\mathrm{TSC}^{-/-}$mice without any changes in lung telomere length (Supplemental Figure 10).

\section{Discussion}

Our data support a major role for mTOR activation in driving lung cell senescence and lung alterations in COPD. Both mTORC1 and mTORC2 were activated in lungs and cells from patients with COPD, and lung cell senescence was strongly associated with mTOR activity. Rapamycin-induced inhibition of mTOR signaling in lung cells from patients with COPD improved replicative capacity and inhibited the SASP. In mice with deletion of TSC1 - a negative regulator of mTORC1 - in targeted lung cells, mTOR activation in these cells was sufficient to induce cell senescence and inflammation. Moreover, TSC1 deletion in P-ECs or AECs led to the spontaneous development of lung emphysema in the absence of additional stimuli. These lung alterations were prevented by in vivo rapamycin treatment. Our findings, thus, support a causal relationship between mTOR activation, cell senescence, and lung alterations in COPD, thereby identifying the mTOR pathway as a new therapeutic target in COPD.

Although mTOR signaling has an established role in several age-related diseases (27), its contribution to lung diseases remains unclear. Activation of mTOR contributes to proliferative lung diseases such as lung cancer and pulmonary hypertension (28-31), but its potential involvement in degenerative lung diseases such as COPD has not been specifically investigated. Our evidence of mTOR overactivity and increased cell senescence in COPD, combined with the efficacy of rapamycin in decreasing both mTOR activity and cell senescence, point to mTOR activation as a major driver of cell senescence in lungs from patients with COPD.

In COPD, increased cell turnover due to accelerated lung-tissue repair and subsequent telomere shortening promotes replicative cell senescence $(11,32)$. Concomitantly, exposure to oxidative stress due to cigarette smoke and chronic inflammation may promote premature cell senescence $(5,33)$. Consistent with this possibility, mTOR was required for oxidative stress-induced cell senescence to occur (17, 34), and mTOR activation was associated with oxidative stress in peripheral blood mononuclear cells from patients with $\operatorname{COPD}(35,36)$. Previous studies also documented a role of oxidative stress and mTOR activation in response to cigarette smoke exposure (37), and some effects of rapamycin on cell senescence have been related to changes in oxidative stress (17). Although this aspect was not specifically assessed in our study, we found that the potent antioxidant NAC neither delayed cell senescence nor altered the SASP.

One hypothesis is that the protective effect of rapamycin against cell senescence is mediated by SASP inhibition. The inflammatory cytokines IL-6, IL-8, and IL-1 $\beta$ released as part of the SASP are well-known inducers of cell senescence $(38,39)$. Rapamycin was recently shown to suppress IL-6 and IL-8 expression 

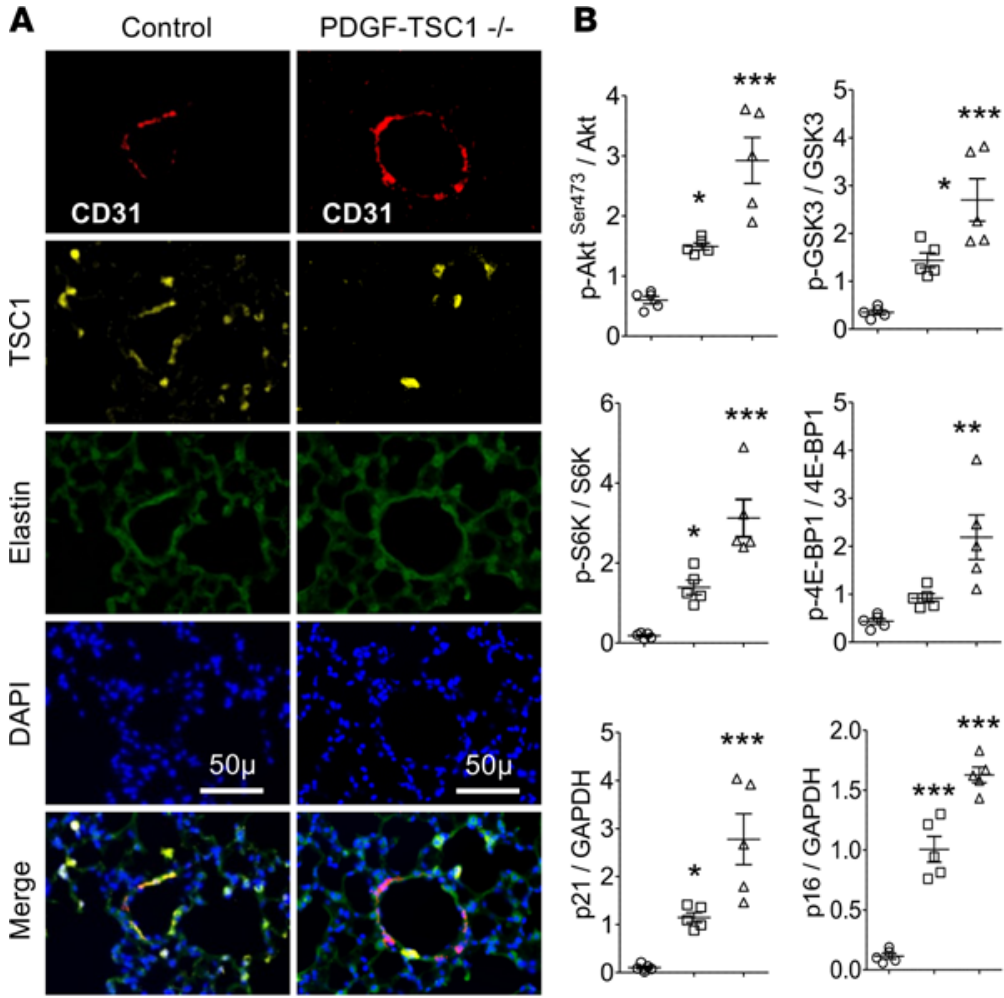

$\circ$ Control

- 3 months PDGF-TSC1 -/

$\triangle 6$ months PDGF-TSC 1 -
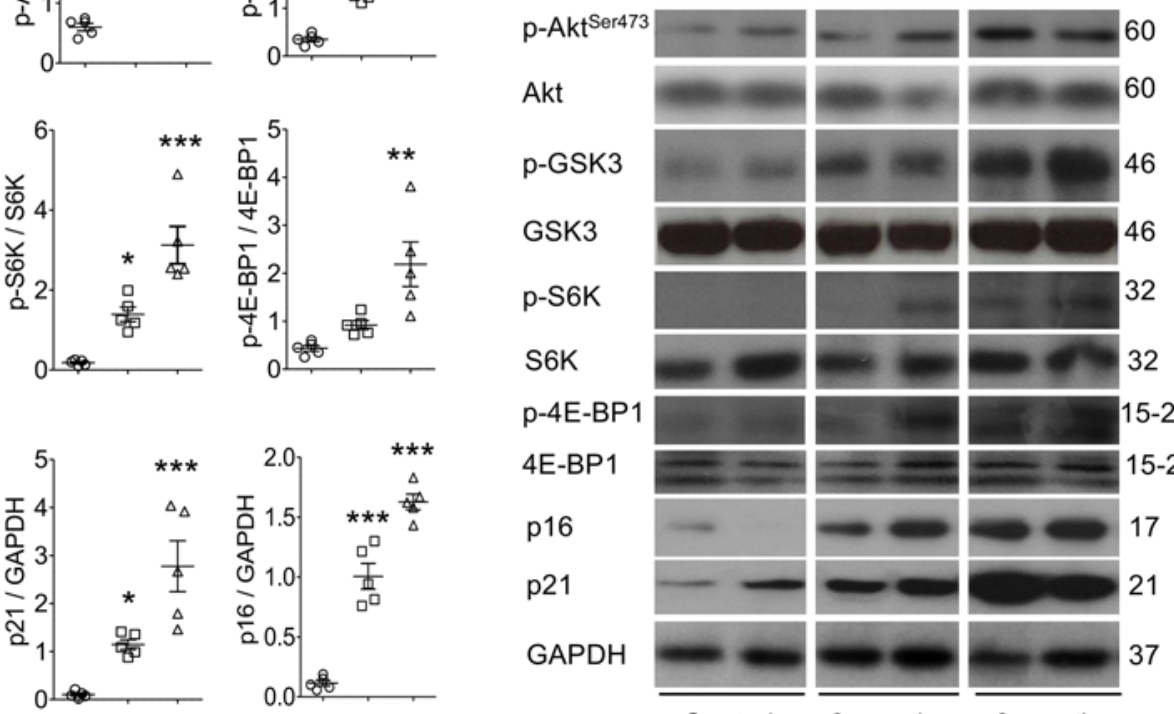

(kDa)
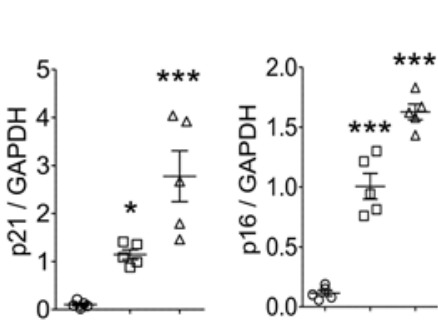

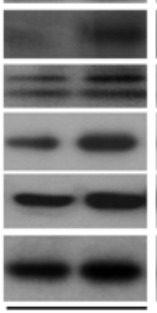

3 months PDGF-

TSC1 -/-
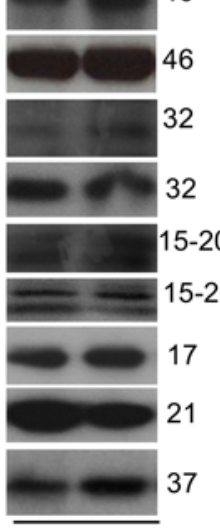

6 months PDGF-

TSC1 -/-

Figure 6. Effects of mTOR overactivation in PDGF-TSC1 ${ }^{-/-}$mice. TSC1 deletion was induced by i.p. tamoxifen in PDCF-TSC1 ${ }^{-1-}$ mice, which were investigated 3 and 6 months later comparatively with vehicle-treated mice. (A) Representative photographs of CD31-positive endothelial cells stained for TSC1 in control (left) and PDGF-TSC1 ${ }^{-1-}$ (right) mice. Scale bars: $50 \mu \mathrm{m}$. (B) Lung levels of Akt phosphorylated at Ser473 (Akt-Ser473), glycogen synthase kinase 3 (GSK3), S6 kinase (S6K), 4E-binding protein 1 (4E-BP1), p21, and p16 proteins in PDCF-TSC1-- mice 3 and 6 months after starting tamoxifen treatment, compared with vehicle-treated control mice. ${ }^{*} P<0.05$, ${ }^{* *} P<0.01,{ }^{* *} P<0.001 \mathrm{vs}$. control mice (1-way ANOVA).

in senescent human cells by acting on the translation of IL-1 $\beta$, a master regulator of the NF- $\kappa \mathrm{B}$ transcription factor (40). In our study, however, blocking the release of IL-6, IL-8, and IL-1 $\beta$ via NF- $\kappa$ B inhibition blocked the SASP without affecting cell senescence. Thus, rapamycin did not act via SASP inhibition.

Another hypothesis is that SASP inhibition was a consequence of cell senescence inhibition by rapamycin. However, curative rapamycin decreased the expression of SASP components without affecting the $\beta$-Gal-positive cell count. Thus, rapamycin had distinct effects on cell senescence and on the SASP in COPD. In previous studies (40), the effects of rapamycin were mimicked by genetic or pharmacological inhibition of S6K. We cannot conclude about the role of S6K in our study, since we only assessed the effects of pharmacological S6K1 inhibition. One may also question the significance of the relatively low levels of SASP components released by cultured cells. This point was not specifically examined in the present study, although, in previous studies, we found a strong relationship between SASP components measured in lung cells and lung homogenates from patients with COPD and controls (11).

TSC1 deletion in our 3 mouse strains - SM22-TSC1 ${ }^{-/-}, \mathrm{PDGF}_{-\mathrm{TSC}}{ }^{-/-}$, and SPC-TSC1 $1^{-/-}$- increased the lung levels of phosphorylated substrates of both mTORC1 and mTORC2, in line with earlier data (24). SM22-TSC1 ${ }^{-1-}$ mice had early senescence of cultured PA-SMCs, which was partly corrected by rapamycin, and high lung p16 and p21 protein levels. Thus, TSC1 deletion replicated the activation of both mTORC1 and mTORC2 that we found in cells and lungs from patients with COPD. Moreover, inducing TSC1 deletion in conditionally TSC1-depleted mice led to rises in lung p16 and p21 levels, which were closely associated with mTORC1 and mTORC2 activation. Thus, inducing mTOR activation in targeted lung cells was sufficient to induce cell senescence in vivo.

To assess whether cell senescence induction replicated some of the lung alterations seen in COPD, we investigated lung functional and structural parameters in PDGF-TSC1 ${ }^{-/-}$and SPC-TSC1 ${ }^{-/-}$mice. We targeted 
A

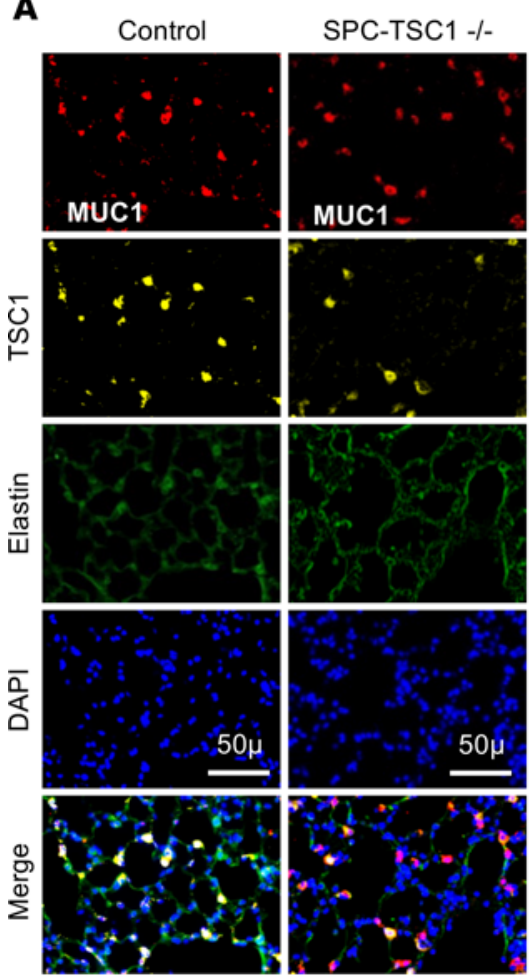

B
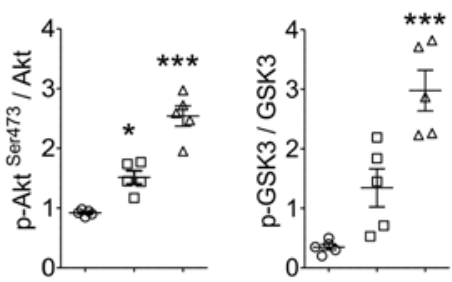

- Contro

- 3 months SPC-TSC 1 -/-

$\triangle 6$ months SPC-TSC1 -/-
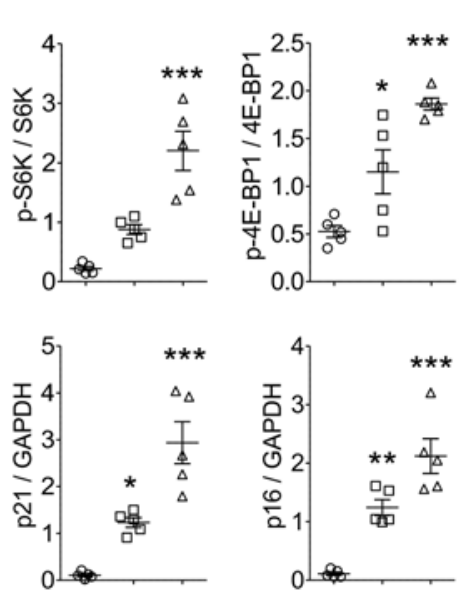

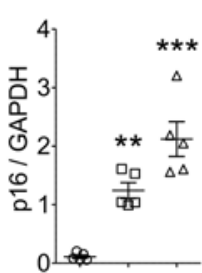

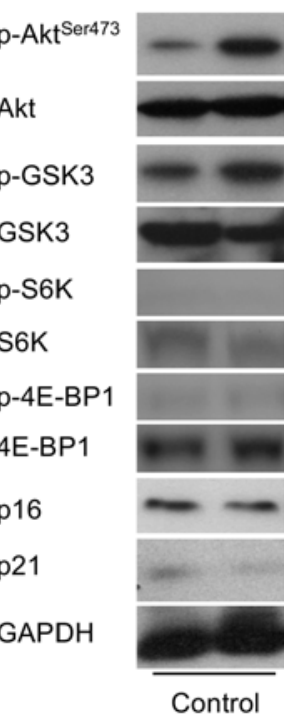
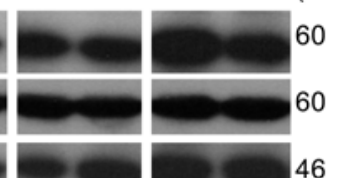

(kDa)
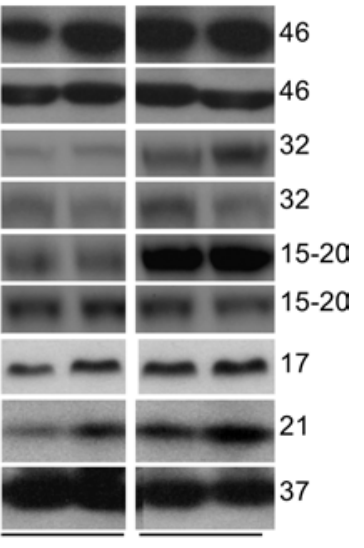

3 months SPCTSC1-/-

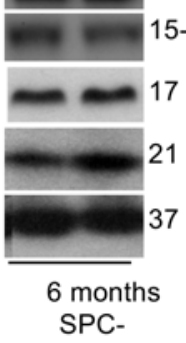

TSC1-/-

Figure 7. Effects of mTOR overactivation in SPC-TSC1 ${ }^{-1-}$ mice. TSC1 deletion was induced by adding doxycycline to the drinking water of SPC-TSC1 ${ }^{-/-}$mice, which were investigated 3 and 6 months later comparatively with vehicle-treated mice. (A) Representative photographs of MUC1-positive alveolar epithelial cells stained for TSC1 in control (left) and SPC-TSC1-/- (right) mice. Scale bars: $50 \mu \mathrm{m}$. (B) Lung levels of Akt phosphorylated at Ser473 (Akt-Ser473), glycogen synthase kinase 3 (CSK3), S6 kinase (S6K), 4E-binding protein 1 (4E-BP1), p21, and p16 proteins in SPC-TSC1 ${ }^{-/-}$mice 3 and 6 months after starting doxycycline treatment, compared with vehicle-treated control mice. ${ }^{*} P<0.05,{ }^{*} P<0.01,{ }^{* *} P<0.001 \mathrm{vs}$. control mice (1-way ANOVA).

P-ECs or AECs in these experiments because dysfunctional P-ECs or AECs are major contributors to the pathogenesis of lung emphysema $(41,42)$. Both PDGF-TSC1 ${ }^{-/}$and SPC-TSC1 $1^{-/}$mice developed lung emphysema 3 months after TSC1 deletion, concomitantly with elevations in lung p16 and p21 levels. Interestingly, these mice also developed pulmonary hypertension, a finding consistent with the reported association between pulmonary hypertension and the development of lung emphysema in mouse models (42). One limitation in our assessment of lung emphysema is that lung volume was not measured. This point may have led to overestimation of the results (43). However, the close association between the development of lung emphysema and pulmonary hypertension in our models is consistent with the pathophysiological mechanisms occurring during cigarette smoke exposure. (42). These changes were also associated with macrophage infiltration of the lung parenchyma and perivascular areas, as well as with increases in lung cytokine levels. A further argument supporting a role for mTOR activation in driving lung alterations was that rapamycin treatment, started concomitantly with parenteral tamoxifen, prevented the development of lung emphysema and pulmonary hypertension in PDGF/TSC1 $1^{-/}$mice. Moreover, in accordance with results from COPD lung cells, chronic rapamycin treatment also reduced the increased lung cytokine levels in PDGF/TSC1 $1^{-/}$mice. These results are consistent with previous studies showing that rapamycin decreased alveolar inflammation and prevented emphysema in WT mice exposed to cigarette smoke (37). Taken together, these results in mice show that mTOR pathway activation is sufficient to drive cell senescence, generate the SASP, and induce emphysema and pulmonary hypertension, even in young mice.

Because mTOR is an essential negative regulator of autophagy $(44,45)$, mTOR activation may decrease autophagy, thereby promoting cell senescence. The decrease in autophagy proteins in lungs from TSC1-KO mice is consistent with this possibility. Moreover, lung SIRT1 was reduced in PDGF-TSC1 $1^{-/}$and SPC$\mathrm{TSC1}^{-/-}$mice, a finding also reported in mice developing emphysema due to cigarette smoke exposure (46). SIRT1 mediates the deacetylation of ATG proteins and transcription factors, including the FOXO3 family, 
A
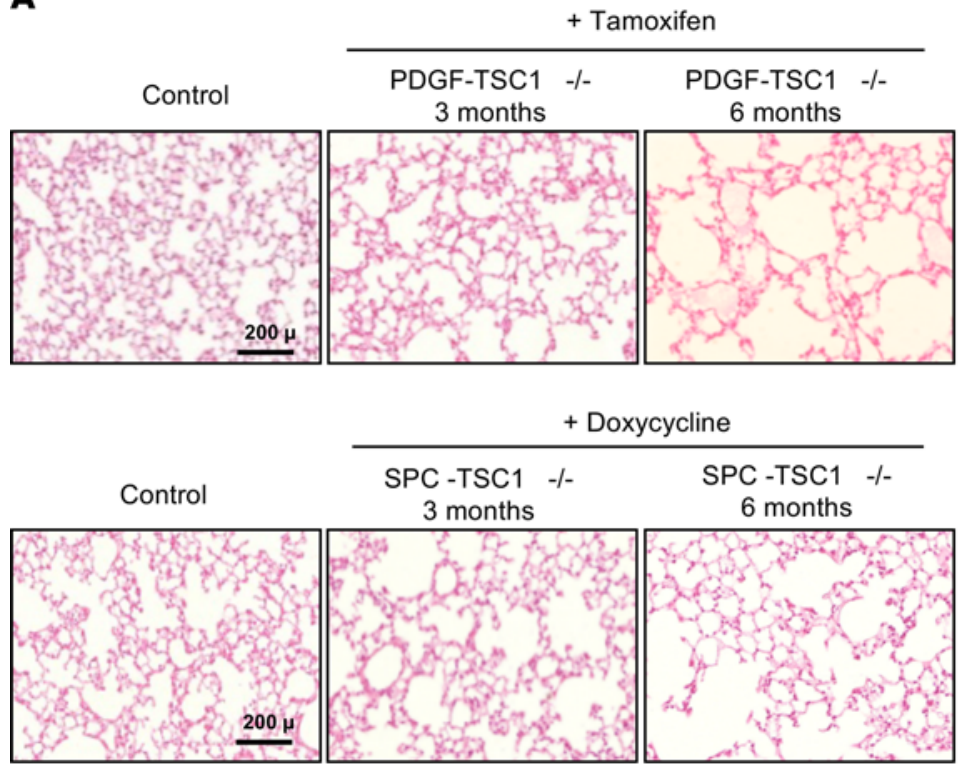
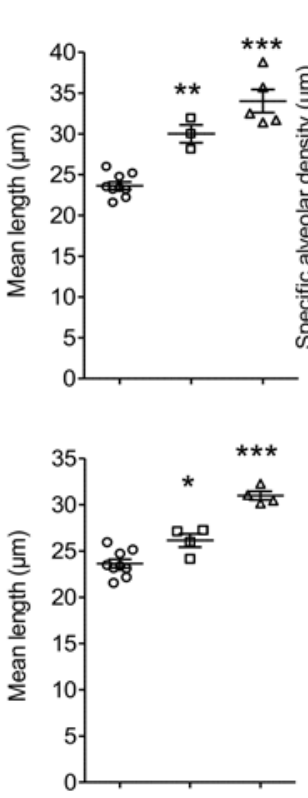
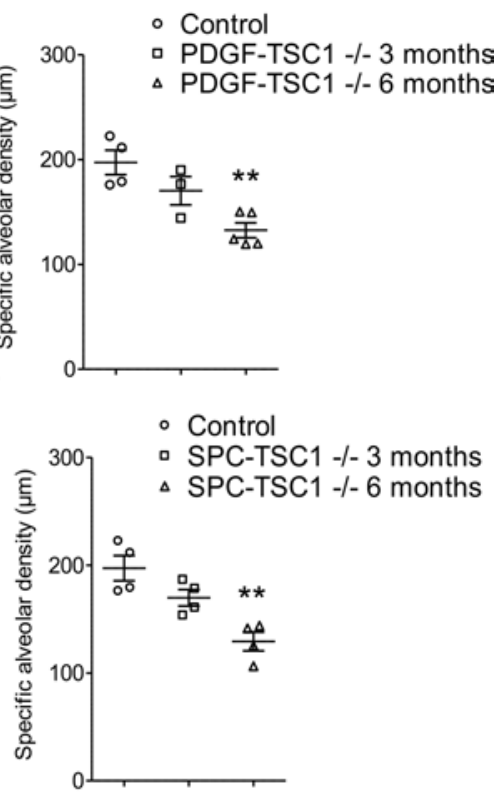

B
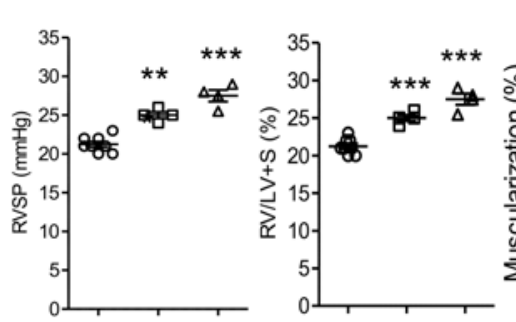

Control

- PDGF-TSC1 -/- 3 months $\triangle$ PDGF-TSC1 -/- 6 months

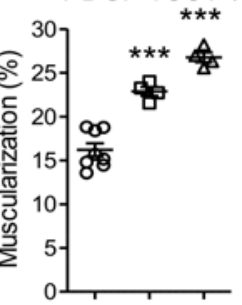

+ Tamoxifen
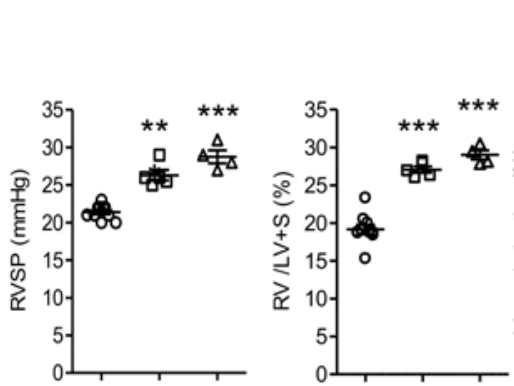

- Control

- SPC-TSC1 -/- 3 months

$\triangle$ SPC-TSC1 -/-6 months

\section{PDGF -TSC1 -/- PDGF -TSC1 -/-} 3 months

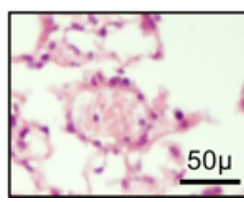

6 months

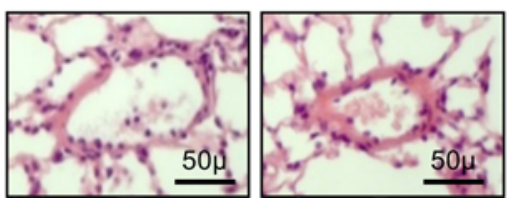

Control

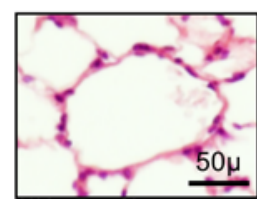

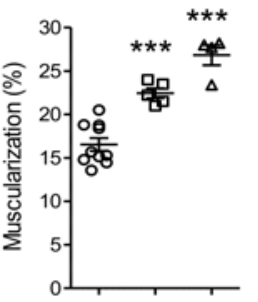

+ Doxycycline

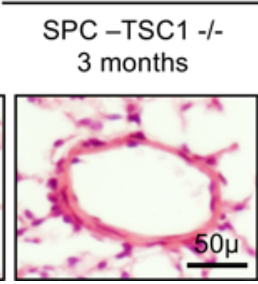

SPC -TSC1 -/6 months

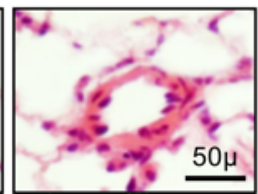

Figure 8. Effects of mTOR overactivation in SPC-TSC1 ${ }^{-/-}$and PDGF-TSC1 ${ }^{-/-}$mice. TSC1 deletion was induced by i.p. tamoxifen in PDCF-TSC1 ${ }^{-/-}$mice and by treatment with doxycycline in drinking water in SPC-TSC1 ${ }^{-1-}$ mice. The mice were investigated 3 and 6 months later and were compared with vehicle-treated mice. (A) Development of lung emphysema in PDCF-TSC1 ${ }^{-/-}$and SPC-TSC1 ${ }^{-/-}$mice. From left to right, representative lung sections stained with H\&E and showing emphysema lesions in PDCF-TSC1 ${ }^{-1-}$ and SPC-TSC1 $1^{-/-}$mice, mean linear intercept of alveolar septa, and air space. Scale bars: $200 \mu \mathrm{m}$. (B) Development of pulmonary hypertension in PDCF-TSC1 ${ }^{-/-}$and SPC-TSC1-/- mice. Right ventricular systolic pressure (RVSP), right ventricular/left ventricular + septum weight ratio $(R V / L V+S)$, and muscularization of pulmonary vessels (percent of muscularized vessels over the total number of pulmonary vessels). Representative pulmonary vessels in PDGF-TSC1 ${ }^{-1-}$ and SPC-TSC1 $1^{-1-}$ mice compared with control mice. ${ }^{*} P<0.05,{ }^{* *} P<0.01,{ }^{* * *} P<0.001$ vs. control mice (1-way ANOVA). Scale bars: $50 \mu \mathrm{m}$.

and stimulates autophagy $(47,48)$. It is therefore possible that, in mice with TSC1 deletion, both mTOR activation and reduction in SIRT1 altered autophagy. Moreover, SIRT1 has been shown to protect against emphysema via FOXO3-mediated reduction of premature senescence in mice (46). Further studies are therefore warranted to explore the potential relationship between the mTOR pathway, the SIRT1-FOXO axis, and autophagy in the process of lung cell senescence and development of emphysema.

The mechanisms at the origin of mTOR activation in COPD remain to be clarified. In previous studies, Rtp801, a stress-related protein that inhibits mTOR and enhances oxidative stress, was suggested as a potential molecular sensor linking cigarette smoke-induced lung injury to the mTOR pathway (37). However, Rtp801 is overexpressed in human lungs with emphysema, and Rtp801-KO mice are protected against acute cigarette 
A

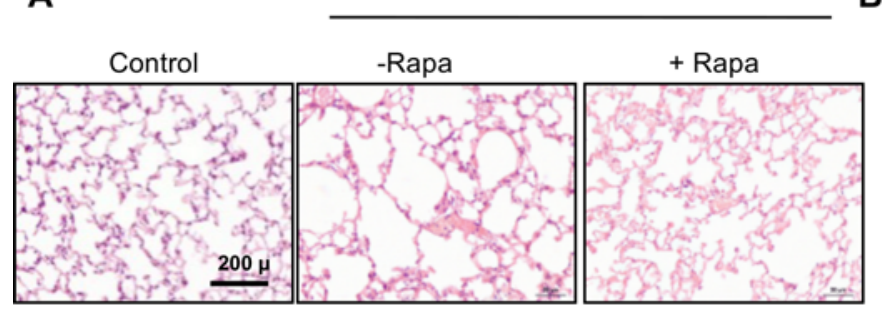

C

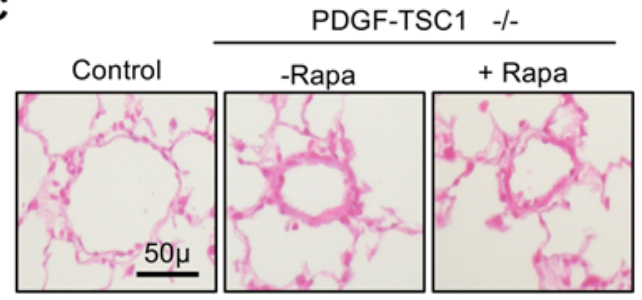

B

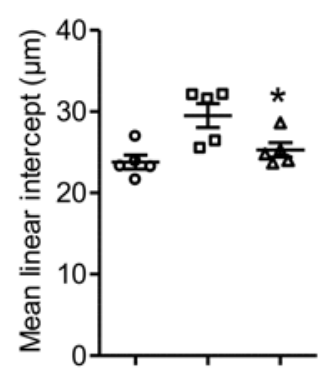

- Control

- PDGF-TSC1 -/- + Vehicle

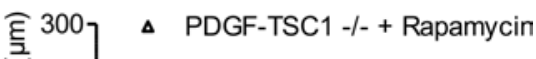

D

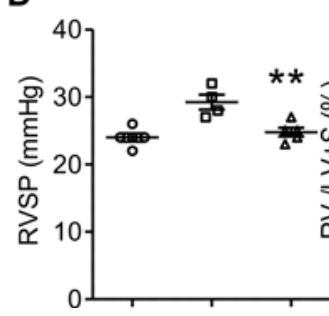

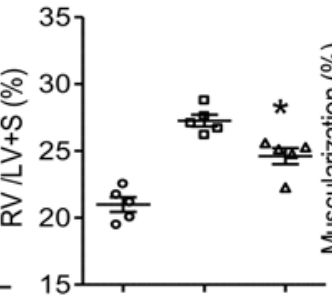

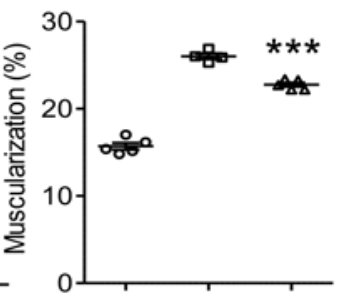

Figure 9. Effects of rapamycin-treated mice on lung emphysema and pulmonary hemodynamics in PDGF-TSC1-/-. Rapamycin was given in drinking water $(2.5 \mathrm{mg} / \mathrm{ml})$ and by oral gavage ( 2.5 or $5 \mathrm{mg} / \mathrm{kg}$ every other day) concomitantly with tamoxifen for 3 months. (A) Representative H\&E-stained lung sections showing emphysema lesions in PDGF-TSC1 ${ }^{-1-}$ mice treated with rapamycin or vehicle, compared with control transgenic mice not treated with tamoxifen. Scale bar: $200 \mu \mathrm{m}$. (B) Mean linear intercept of alveolar septa and air space. (C) Representative lung sections showing pulmonary vessel remodeling. Scale bar: $50 \mu \mathrm{m}$. (D) RVSP, RV/(LV/S), and pulmonary-vessel muscularization. ${ }^{*} P<0.05,{ }^{* *} P<0.01,{ }^{* * *} P<0.001 \mathrm{vs}$. vehicle-treated PDCF-TSC1 ${ }^{-1-}$ mice, by 1-way ANOVA.

smoke-induced lung injury (37). It is difficult to relate these findings to changes in mTOR signaling, since the same study showed that rapamycin treatment protected WT mice against cigarette smoke-induced lung emphysema. In previous studies, cigarette smoke exposure was shown to induce Akt hyperphosphorylation of human lung ECs (49). In lungs from patients with COPD and TSC1-depleted mice, we found an increase in pAkt Ser473 and a reduction in $\mathrm{pAkt}^{\mathrm{Th} 308}$. This finding points to increased activity of mTOR complexes, and not of PI3K, at the origin of increased mTOR signaling. Further studies are therefore needed to clarify mechanisms responsible for lung mTOR activation in COPD.

Taken together, our findings support a causal role for mTOR activation-induced cell senescence in lung pathology. That cell senescence may lead to lung emphysema is also strongly indicated by the occurrence of severe lung emphysema in some patients with telomere gene mutations (50). In most cases, COPD develops in the absence of telomere gene mutations, although it is associated with telomere shortening (11, 32). Patients with COPD may also have distinct phenotypes according to the severity of emphysema and chronic bronchitis (4). In previous studies, including ours, cell senescence criteria correlated with the emphysema score $(11,51)$. It is therefore possible that lung mTOR activity, together with cell senescence criteria, may vary according to the phenotype of patients with COPD. In our mice with TSC1 deletion and activation of the mTOR pathway, lung emphysema developed without telomere shortening. These results strongly indicate that various lung stresses in COPD converge to the mTOR pathway to induce premature cell senescence. Targeting the mTOR pathway to inhibit cell senescence or to eliminate SASP components may therefore hold therapeutic promise in COPD.

\section{Methods}

Experimental design. In the first part of the study, we used lung specimens and derived cultured cells, namely PA-SMCs and P-ECs from patients with COPD and age- and sex-matched control smokers, to assess mTOR signaling elements and cell senescence biomarkers. Cultured cells from patients and controls were then subjected to repeated cell passages to determine their replicative senescence threshold and to measure cytokines released as part of the SASP. To test the hypothesis that mTOR pathway activation drives the cell senescence process in COPD, we investigated whether inhibiting mTOR by rapamycin delayed the onset of cell senescence and suppressed the SASP. In complementary studies, we evaluated whether cell senescence in COPD was affected by inhibiting the synthesis of inflammatory 
A
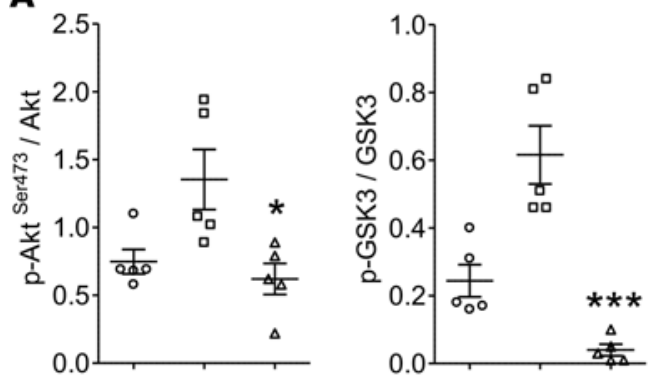

- Control

- PDGF-TSC1-/-

$\triangle$ PDGF-TSC1-/- + Rapamycin
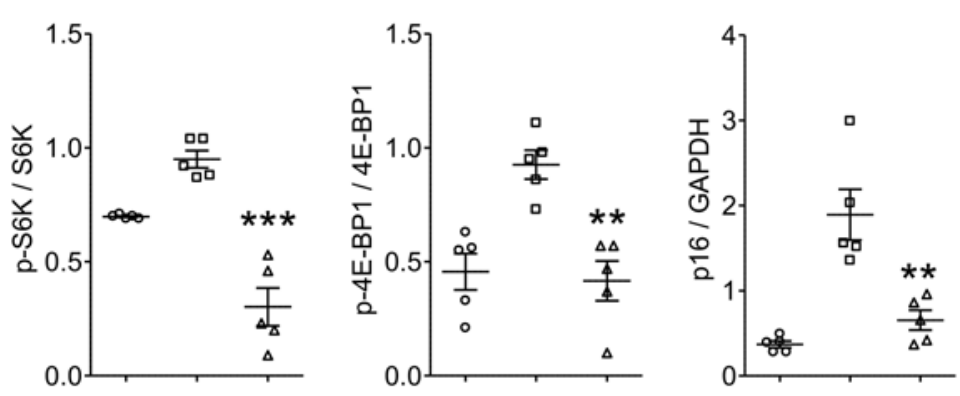

p-Akt ${ }^{\text {Ser473 }}$
Akt

p-GSK3

GSK3

p-S6K

(kDa)

60
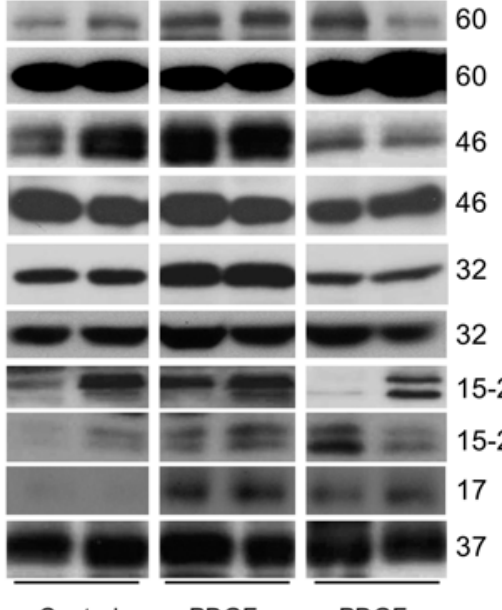

60

46

46

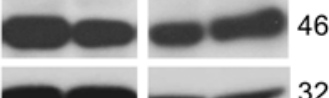

S6K

p-4E-BP1

4E-BP1

p16

GAPDH
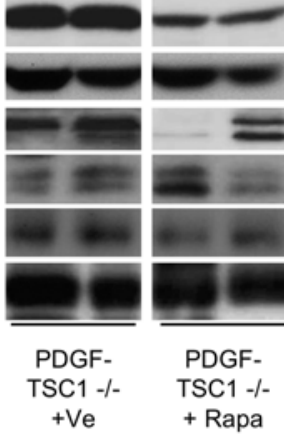

32

\section{B}
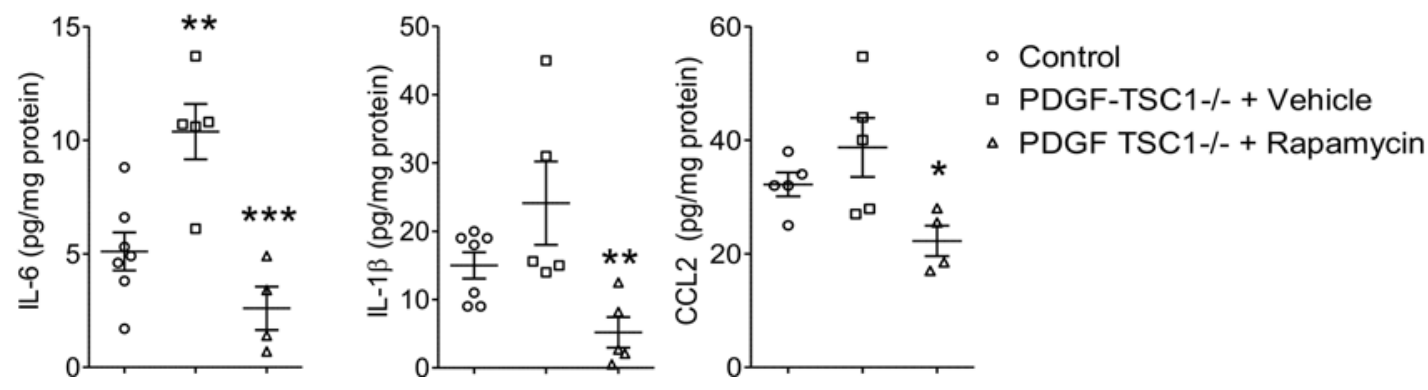

Figure 10. Effects of rapamycin treatment on lung protein levels in PDGF-TSC1-1- mice. (A) Lung levels of phosphorylated Akt-Ser473, GSK3, S6K, and 4E-BP1 normalized for total protein and lung levels of p16 protein, with their corresponding gels in the right panel. (B) Lung levels of the cytokines IL-6, IL-1B, and CCL2. ${ }^{*} P<0.05,{ }^{* *} P<0.01,{ }^{* *} P<0.001$ vs. vehicle-treated PDGF-TSC1-/- mice, by 1-way ANOVA.

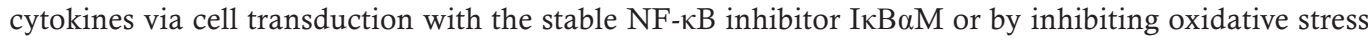
via cell treatment with NAC.

The second part of the study investigated whether mTOR activation in transgenic mouse models was sufficient to induce lung cell senescence and mimic the lung alterations of COPD. To this end, we generated mice with deletion of TSC1, a negative mTORC1 regulator, in targeted cells (i.e., PA-SMCs, P-ECs, or AECs). We performed in vitro studies to investigate the susceptibility of cultured PA-SMCs from SM22/ $\mathrm{TSC1}^{-/-}$mice to replicative senescence and the effects of rapamycin treatment. In mice with conditional TSC1 deletion in P-ECs or AECs, we assessed whether mTOR activation was associated with lung cell senescence and the lung alterations seen in COPD, namely lung emphysema, pulmonary hypertension, and inflammation. In addition, we evaluated whether these lung alterations were prevented by in vivo rapamycin treatment.

Measurements and characteristics of participants. Human lung tissues were obtained from 60 patients who underwent lung resection surgery at the Institut Mutualiste Montsouris. Among them, 30 had COPD and 30 were control smokers matched to the COPD patients on age and sex (Supplemental Table 1). Inclusion criteria for the patients with COPD included a minimum of a 10 pack-year smoking history and a ratio of forced expiratory volume in 1 second $\left(\mathrm{FEV}_{1}\right)$ over forced vital capacity $(\mathrm{FVC})<70 \%$. The controls had to have a smoking history greater than 5 pack-years, an $\mathrm{FEV}_{1} / \mathrm{FVC}$ ratio greater than $70 \%$, and no evidence of chronic cardiovascular, hepatic, or renal disease. None of the patients had a history of cancer chemotherapy. Lung tissue samples collected during surgery were used for in situ immunohistochemical studies, protein level determinations, and cultures of derived PA-SMCs and P-ECs. Cells were cultured from a subset of 14 patients and 16 controls (Supplemental Table 2). 
Studies of lung samples from patients with COPD and controls. Lung samples from patients with COPD and controls were used for Western blot measurements of Akt phosphorylated at serine 473 (p-Akt ${ }^{\text {Ser473}}$ ), Akt, p-GSK3, GSK3, p-S6K, S6K, p-4E-BP1, and 4E-BP1 (all Cell Signaling Technology). The results were normalized for the respective total protein content. The senescence biomarkers p16 and p21 were assayed by Western blotting, and the results were normalized for GAPDH levels. Lung immunohistochemical studies were performed to determine the location of p16-positive senescent cells within pulmonary vessel walls (in PA-SMCs stained for $\alpha$-smooth muscle actin [ $\alpha$-SMA] and P-ECs stained for von Willebrand factor [vWF]) and alveolar septa (in AECs) stained for MUC1 (52).

Cultured cells from patients with COPD and controls. Cultured P-ECs and PA-SMCs were collected from the lungs of patients with COPD and controls as previously described $(11,13)$. Cells at early passages were used to measure p-Akt ${ }^{\text {Ser473 }}$, Akt, p-GSK3, GSK3, p-S6K, S6K, p-4E-BP1, 4E-BP1, p16, and p21 proteins by Western blotting and to determine the percentage of cells with $\beta$-Gal activity. These measurements were performed in cells deprived of serum for 24 hours (ECs) or for 48 hours (SMCs) as previously described $(11,13)$. The cells were then subjected to repeated cell passages to determine their replicative senescence threshold and the total number of cell PDL. At each passage, when the cells reached $80 \%-90 \%$ confluence, they were counted with trypan blue using a hemocytometer. The onset of cell replicative senescence was defined based on cessation of cell division, labeling for SA- $\beta$-Gal, and cell morphology criteria. The PDLs were calculated as follows: PDL $=\left(\log _{10} \mathrm{Y}-\log _{10} \mathrm{X}\right) / \log _{10}{ }^{2}$, where $\mathrm{X}$ is the initial number of seeded cells and $Y$ the final number of cells $(11,17)$.

To assess the effects of mTOR inhibition on replicative cell senescence, cells from patients with COPD and controls were continuously cultured in a medium containing either vehicle or rapamycin (10 $\mathrm{nM})$. In different experiments, cells were continuously treated with the S6K1 inhibitor PF478671 (1 and $5 \mu \mathrm{M}$ ) or with the antioxidant NAC $(0.2$ or $1 \mathrm{mM})$, and the effects were evaluated comparatively with those of rapamycin. Substrates of $\mathrm{mTORC} 1$ and $\mathrm{mTORC} 2$ were measured in cells from patients with COPD and controls treated as described above.

In specific experiments, cells were infected with a retroviral vector expressing I $\mathrm{B} \alpha \mathrm{M}$ resistant to phosphorylation and degradation, the native I $\mathrm{B} \alpha$ gene, or a control vector, as previously described (23). We then compared the effects of I $\mathrm{B} \alpha \mathrm{M}$ transduction with those of the control vector. The protocols used to transfect virus-producing GP293 cells and infect target cells have been described previously (53). The infection protocols were designed to cause infection of nearly all the cells, as demonstrated by the fluorescence observed after infection with a GFP-expressing retroviral vector (not shown).

To measure the secretion of the cytokines IL- 6 , IL-8, and CCL2, cells were rinsed twice in PBS $1 \times$ and placed in reduced media containing $0.2 \%$ FCS without heparin and EC growth supplement. After 24 hours of incubation, the conditioned media were collected and used to quantify IL-6, IL-8, and CCL2 using Quantikine ELISA kits (R\&D systems) according to the manufacturer's instructions.

Senescence associated $\beta$-Gal staining. At each passage, cells at $60 \%$ confluence were fixed with $2 \%$ formaldehyde (MilliporeSigma) and $0.2 \%$ glutaraldehyde (MilliporeSigma) for 10 minutes. Then, the cells were washed with PBS and stained in a titrated pH 6 solution containing $40 \mathrm{mM}$ citric acid, $150 \mathrm{mM} \mathrm{NaCl}, 2$ $\mathrm{mM} \mathrm{MgCl} 2,5 \mathrm{mM}$ potassium ferrocyanide, and $1 \mathrm{mg} / \mathrm{ml} \mathrm{X-Gal}$.

Mice. Adult male mice (C57B1/6J) were obtained from JANVIER LABS. To study the effects of mTOR activation in targeted cells, we generated mice with constitutive or conditional deletion of the tuberous sclerosis complex heterodimer TSC1 (a negative regulator of mTORC1), in PA-SMCs, P-ECs, or AECs. $\mathrm{TSC}^{\mathrm{LoxP} / \mathrm{LoxP}}$ mice were crossed with mice expressing Cre recombinase under the control of the SM22 $\alpha$ promoter (SM22/ $\mathrm{TSC}^{-/-}$mice) to target SMCs, mice expressing a tamoxifen-inducible form of Cre recombinase under the control of the $\mathrm{PDGFb}$ promoter ( $\mathrm{PDGFb}-\mathrm{iCreER} \mathrm{T}^{\mathrm{T}} / \mathrm{TSC}^{\mathrm{LoxP} / \mathrm{LoxP}}$ mice) to target ECs, or mice expressing a doxycycline-inducible form of Cre recombinase under the control of the surfactant protein C (SPC-rtTA/[tetO] $]_{7}$ CMV-Cre mice) promoter (SPC/ $\mathrm{TSC}^{\text {LoxP/LoxP }}$ mice) to target AECs. $\mathrm{PDGFb}$-Cre mice were a gift from M. Fruttiger (National Institute of Medical Research, MRC, London, United Kingdom) (25). SPC-rtTA/(tetO) ${ }_{7}$-CMV-Cre mice were a gift from J.A. Whitsett (Cincinnati Children's Hospital Medical Center, Division of Pulmonary Biology, Cincinnati, Ohio, USA) (26). In PDGF/ TSC1 $1^{\text {LoxP/LoxP }}$ mice, TSC1 deletion was induced by i.p. tamoxifen $0.22 \mathrm{mg} / \mathrm{kg} /$ day for 5 consecutive days. Conditional TSC1 deletion in SPC-TSC $1^{\mathrm{LoxP} / \mathrm{LoxP}}$ mice was induced by treatment with doxycycline in drinking water $(1 \mathrm{mg} / \mathrm{ml})$ for various durations. Control mice were corresponding transgenic mice not treated with tamoxifen or doxycycline. For SM22/ $\mathrm{TSC}^{-/-}$mice, controls were littermates, as previously described 
(24). To assess the in vivo effect of rapamycin treatment, we treated mice preventively with rapamycin, in drinking water $(2.5 \mathrm{mg} / \mathrm{l})$ and by simultaneous gavage with $2.5 \mathrm{mg} / \mathrm{kg}$ twice a week.

Animal preparation, assessment of lung emphysema and pulmonary hypertension, and lung tissue analysis. Mice were anesthetized with an i.p. injection of ketamine $(60 \mathrm{mg} / \mathrm{kg})$ and xylazine $(10 \mathrm{mg} / \mathrm{kg})$. RVSP was measured as described previously (54). The RV was dissected from the left ventricle plus septum (LV+S), and these dissected samples were weighted to determine the Fulton index $(\mathrm{RV} /[\mathrm{LV}+\mathrm{S}])$. The right lung was quickly removed and divided into 2 parts, which were immediately snap-frozen in liquid nitrogen and then stored at $-80^{\circ} \mathrm{C}$ until total RNA and protein extraction for biological measurements (real-time PCR, Western blot, and ELISA). Immunostaining, Western blot, ELISA, and real-time PCR were performed as described previously $(24,54)$. The left lungs were fixed by intratracheal infusion of $4 \%$ paraformaldehyde aqueous solution (MilliporeSigma) at a transpleural pressure of $30 \mathrm{cmH}_{2} \mathrm{O}$. For morphometry studies, 5 $\mu \mathrm{m}$-thick sagittal sections along the greatest axis of the left lung were cut in a systematic manner and stained with $\mathrm{H} \& \mathrm{E}$. Alveolar and vascular morphometry studies were performed by an observer blinded to treatment and genotype, as described elsewhere $(54,55)$.

Lung emphysema was assessed using a combination of the point-counting method for alveolar surface density and the mean-linear-intercept (MLI) method described by Knudsen et al. (56). Light microscope fields were quantitated at an overall magnification of 400, using a 42-point and 21-line grid. We examined 20 fields/animal (10 per lung), using a systematic sampling method from a random starting point. To correct area values for shrinkage associated with fixation and paraffin processing, we used the factor of 1.22 calculated during a previous study (54). MLI was also determined by computer-assisted morphometry using a macro designed with ImageJ software (Molecular Devices). Five black and white digital photomicrographs were acquired from the cranial, medial, and caudal regions of each slide at 400× magnification, excluding areas where large bronchi or vessels predominated, to obtain 15 images per lung. Emphysema was then quantified by measuring the mean alveolar chord length with image analysis software (ImageJ System) at $5-\mu \mathrm{m}$ intervals. This automated analysis was made vertically and horizontally on each photomicrograph. The mean alveolar chord length was obtained by averaging the measurements. All morphometric data were expressed as absolute values.

For vascular morphometry, each vessel was categorized as nonmuscular (no evidence of vessel wall muscularization), partially muscular, or fully muscular. The percentage of muscular pulmonary vessels was determined by dividing the sum of partially and fully muscular vessels by the total number of vessels in the relevant group of animals, as described previously (54).

Immunofluorescence. Paraffin-embedded sections were deparaffinized using xylene, and a graded series of ethanol dilutions was then incubated in citrate buffer $\left(0.01 \mathrm{M}, \mathrm{pH}\right.$ 6) at $90^{\circ} \mathrm{C}$ for 20 minutes. Tissues were permeabilized with $0.1 \%$ Triton X-100 in phosphate-buffered saline (PBS) for 10 minutes.

For immunofluorescence, paraffin-embedded sections were prepared as described above; cells were fixed for 10 minutes in $1 \%$ formalin in PBS and then permeabilized with $0.05 \%$ Triton X-100 in PBS for 10 minutes. Saturation was achieved using Dako antibody diluents with $10 \%$ goat serum. For double immunostaining, sections were incubated with first primary antibody (diluted in Dako antibody diluents with $3 \%$ goat serum) for 1 hour at $37^{\circ} \mathrm{C}$ in a humidified with chamber, washed 3 times with PBS, and incubated 1 hour with a second primary antibody produced in different host species. After PBS washes, the sections were incubated with 2 secondary antibodies produced in 2 different host species and conjugated with 2 different fluorochromes for 40 minutes at $37^{\circ} \mathrm{C}$ in a humidified chamber. Basically, to avoid interference with green elastin autofluorescence, Red (555) and Far-Red (660) conjugate were used for labeling of lung sections, and Green (488) and Red (555) conjugate were used for double immunolabeling of cells. The following secondary antibodies were used in this study: goat anti-rat IgG, Alexa Fluor 488 conjugate (catalog A-11006); goat anti-rabbit IgG, Alexa Fluor 546 conjugate (catalog A-11010); rabbit anti-mouse IgG, Alexa Fluor 488 conjugate (catalog A-11059); goat anti-mouse IgG, Alexa Fluor 660 conjugate (catalog A-21054); donkey anti-rabbit IgG, Alexa Fluor 488 conjugate (catalog A-21206); goat anti-mouse IgG, Alexa Fluor 555 conjugate (catalog A-21422); goat anti-rat IgG, Alexa Fluor 555 conjugate (catalog A-21434) (all from Invitrogen); goat anti-rabbit IgG, highly cross-adsorbed, CF660C conjugate (catalog SAB4600453); goat anti-chicken IgY (IgG), highly cross-adsorbed (catalogs CF660C, SAB4600457); and goat anti-rat IgG, highly cross-adsorbed (catalogs CF660C, SAB4600455) (all from MilliporeSigma). The sections were protected with coverslips secured with fluorescent mounting medium containing DAPI for nuclear staining. 
Detection of apoptotic cells on lung tissue. Apoptotic cells were identified by detection of double-stranded DNA fragmentation using a TUNEL assay (In Situ Cell Death Detection Kit, TMR red, catalog 12156792910, Roche Diagnostics) according to manufacturer's instructions. Nuclear staining by DAPI was performed as counterstaining.

Extraction and culture of mouse PA-SMCs (mPA-SMCs). mPA-SMCs were obtained from pulmonary arteries by digestion in DMEM (Invitrogen) with collagenase $(0.25 \mu \mathrm{g} / \mathrm{ml})$ and elastase $(80 \mu \mathrm{g} / \mathrm{ml})$ (MilliporeSigma). These cells were then grown at $37^{\circ} \mathrm{C}$ in $5 \% \mathrm{CO}_{2}$ in DMEM supplemented with $15 \% \mathrm{FCS}$. We identified mPA-SMCs as cells exhibiting positive $\alpha$-SMA staining. To assess the effects of mTOR inhibition on PA-SMC senescence, rapamycin (50 nM) or vehicle (DMSO) was added to the cell cultures before and 24 hours after growth-factor exposure.

Telomere length measurement. Telomere length was assessed with quantitative PCR (qPCR). Briefly, the ratio of the telomere repeat copy number to the single gene copy number was determined by use of by use of QuantStudio 6 Flex Real-Time PCR System from Thermo Fisher Scientific in a 384-well format and the comparative Ct method (ratio of the telomere repeat copy number to the single-gene copy number, $2^{-\Delta \Delta \mathrm{Ct}}$ ). Genomic DNA was extracted from PA-SMCs with the QIAamp DNA Kit (Qiagen) and quantified with a spectrophotometer. Each sample (30 ng DNA) was run in triplicate with the SYBR Green method (Invitrogen). The sequences and final concentrations of the primers for the telomere and 36B4 (acidic ribosomal phosphoprotein PO, a single-copy gene used for normalization) were as follows: Tel1, 5'-CGGTTTGTTTGGGTTTGGGTTTGG GTTTGGGTTTGGGTT-3', 300 nmol/1; Tel2, 5'-GGCT TGCCTTA CCCTTACCCTTACCCTTACCCTTACCCT-3', 300 nmol/1; 36B4F, 5'-CAGCAAGTGGGAAGGTGTAATCC-3', $300 \mathrm{nmol} / 1$; and 36B4R, 5'-CCCATTCTATCATCAACGGGTACAA-3', 300 nmol/1.

Telomere length was expressed as the ratio of the telomere repeat copy number over the single-gene copy number (36B4 gene) (32). Triplicate values of each sample had a $\Delta \mathrm{Ct} \mathrm{SD}<0.1$.

Protein extraction and immunoblotting. Total proteins were extracted using RIPA lysis buffer (10 mM

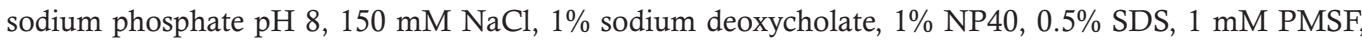
$10 \mathrm{mM}$ NaF, $1 \mathrm{mM}$ sodium orthovanadate, and protease inhibitor cocktail; Roche Diagnostics). Immunoblots were carried out using the indicated antibodies and detected using an enhanced chemiluminescence detection system (GE Healthcare). Densitometric quantification was normalized for the $\beta$-actin level using GeneTools software (Ozyme).

Materials. The mTOR inhibitor rapamycin (Sirolimus, Pfizer) used for animal studies was from the pharmacy of the Henri Mondor Hospital (Créteil, France). Rapamycin used for cell studies and the S6K inhibitor (PF478671) was purchased from MilliporeSigma and dissolved in DMSO. N-acetylcysteine was obtained from MilliporeSigma and dissolved in DMEM. Tamoxifen and doxycycline were purchased from MilliporeSigma.

The following primary antibodies were used: anti- $\beta$-actin ( $\beta$-Act, A5316, MilliporeSigma); anti- $\alpha$ SMA (ab5694, Abcam, and A2547, MilliporeSigma); anti-GAPDH (sc-25778, Santa Cruz Biotechnology Inc.); anti-CDK2A/p16INK4a (p16, ab54210, Abcam; 25084, Abbiotec; and LS-C2744/69800, LSBio); anti-p21 Waf1/Cip1 (p21, 2946, Cell Signaling Technology); anti-Ki67 (KI67, ab66155, Abcam); anti-pAkt (Ser473) (4060, Cell Signaling Technology); anti-p-Akt (T308, Cell Signaling Technology); anti-Akt (9272, Cell Signaling Technology); anti-p-GSK-3 $\beta$ (Ser9) (9331, Cell Signaling Technology); anti-GSK$3 \beta$ (9315, Cell Signaling Technology); anti-p-S6K (2211, Cell Signaling Technology); anti-S6K (2317, Cell Signaling Technology); anti-p-4E-BP1 (9644, Cell Signaling Technology); anti-4E-BP1 (2855, Cell Signaling Technology); anti-MUC1 (ab109185 and ab31998, Abcam); anti-hamartin (TSC1) (ab21632, Abcam); anti-proliferating cell nuclear antigen (PCNA, ab29, Abcam); and anti-VWF (ab6994, Abcam); anti-LC3A/B (D3U4C, Cell Signaling Technology); anti-ATG3 (3415, Cell Signaling Technology); antiATG5 (D5F5U, Cell Signaling Technology); anti-SIRT1 (sc-74504, Santa Cruz Biotechnology Inc.).

Statistics. Data are expressed as mean \pm SEM. Patients with COPD and controls were compared using the unpaired $t$ test for quantitative variables and the $\chi^{2}$ test for categorical variables. Correlations between variables were evaluated using least-square linear regression techniques. One-way or 2-way ANOVA, followed by the Bonferroni's multiple comparison and a 2-tailed Student's $t$ test, was used to compare the means of more than 2 independent groups. $P$ values less than 0.05 were considered significant. Data were analyzed using GraphPad Prism statistical software 5.0 (San Diego, CA).

Study approval. This study was approved by the IRB of the Henri Mondor Teaching Hospital in Créteil, France. All patients and controls signed an informed consent document before study inclusion. Mice were 
used according to institutional guidelines, which complied with national and European regulations. All animal experiments were approved by the IACUC of the French NIH and Medical Research (INSERM) Unit 955, Créteil, France.

\section{Author contributions}

$\mathrm{AH}$ designed and conducted the cell culture and animal studies and interpreted the results. MB designed and conducted the cell culture and animal studies, as well as lung morphometry, and interpreted the results. KK conducted endothelial cell culture studies and interpreted the results. S. Abid designed and conducted the animal studies, lung morphometry, and immunohistochemical analyses and interpreted the results. EM conducted SMC culture studies, retroviral transduction, and ELISA and interpreted the results. LL helped in technical advice and immunohistochemical analyses and interpreted the results. DR performed cell culture studies and Western blot analysis. AP performed cell culture studies and retroviral transduction and interpreted the results. $\mathrm{JH}$ performed cell culture studies and retroviral transduction and interpreted the results. VA gave technical advice and performed EC culture analyses. NV conducted SMC culture studies. PV recruited the patients and conducted organization of the study. BM manages the recruitment of the patients and organization of the study. AA performed cell culture studies and interpreted the results. CL gave technical advice, performed Western blot analyses, and interpreted the results. DV gave technical advice, especially on gene transduction. JB designed the study and gave advice on lung emphysema. GD designed the study and gave advice on cell senescence. MP designed the study and gave advice on mTOR signaling. DB designed the study and gave advice on cell senescence. SM designed the study and interpreted the results. S. Adnot designed the study, interpreted the results, and drafted the manuscript.

\section{Acknowledgments}

We thank Jeffrey A. Whitsett (Cincinnati Children's Hospital Medical Center, Cincinnati, Ohio, USA) for providing the SPC-rtTA/(tetO) ${ }_{7}$-CMV-Cre mice and Marcus Fruttiger (London, United Kingdom) for providing the PDGFb-iCreER ${ }^{\mathrm{T} 2}$ mice. We are grateful to R. Souktani, P. Caramelle, and M. Collery from the conventional animal facility, as well as to X. Decrouy and C. Micheli from the imaging platform facility at IMRB Inserm, Créteil, France

This study was supported by grants from the INSERM, Délégation à la Recherche Clinique de l'AP-HP, Fondation pour la Recherche Médicale (FRM), Legs Poix, and Agence Nationale pour la Recherche ANR R14-086JJ. A. Houssaini is the recipient of a European Respiratory Society Fellowship (LTRF [RESPIRE2 - 2014 - 7130]-2018). The research leading to these results has received funding from the European Respiratory Society

Address correspondence to: Serge Adnot, Hôpital Henri Mondor, Service de Physiologie-Explorations Fonctionnelles, 94010, Créteil, France. Phone: 33.149.812.677; Email: serge.adnot@inserm.fr.

1. Gershon AS, Warner L, Cascagnette P, Victor JC, To T. Lifetime risk of developing chronic obstructive pulmonary disease: a longitudinal population study. Lancet. 2011;378(9795):991-996.

2. Celli BR, MacNee W, ATS/ERS Task Force. Standards for the diagnosis and treatment of patients with COPD: a summary of the ATS/ERS position paper. Eur Respir J. 2004;23(6):932-946.

3. Vanfleteren LE, et al. Clusters of comorbidities based on validated objective measurements and systemic inflammation in patients with chronic obstructive pulmonary disease. Am J Respir Crit Care Med. 2013;187(7):728-735.

4. Han MK, et al. Chronic obstructive pulmonary disease phenotypes: the future of COPD. Am J Respir Crit Care Med. 2010;182(5):598-604.

5. Adnot S, et al. Telomere Dysfunction and Cell Senescence in Chronic Lung Diseases: Therapeutic Potential. Pharmacol Ther. 2015;153:125-134.

6. Armanios M, Blackburn EH. The telomere syndromes. Nat Rev Genet. 2012;13(10):693-704.

7. Muñoz-Espín D, Serrano M. Cellular senescence: from physiology to pathology. Nat Rev Mol Cell Biol. 2014;15(7):482-496

8. Coppé JP, Desprez PY, Krtolica A, Campisi J. The senescence-associated secretory phenotype: the dark side of tumor suppression. Annu Rev Pathol. 2010;5:99-118.

9. Mathon NF, Lloyd AC. Cell senescence and cancer. Nat Rev Cancer. 2001;1(3):203-213

10. Hayflick L. THE LIMITED IN VITRO LIFETIME OF HUMAN DIPLOID CELL STRAINS. Exp Cell Res. 1965;37:614-636.

11. Amsellem V, et al. Telomere dysfunction causes sustained inflammation in chronic obstructive pulmonary disease. Am J Respir Crit Care Med. 2011;184(12):1358-1366.

12. Dagouassat M, et al. The cyclooxygenase-2-prostaglandin E2 pathway maintains senescence of chronic obstructive pulmonary 
disease fibroblasts. Am J Respir Crit Care Med. 2013;187(7):703-714.

13. Noureddine $\mathrm{H}$, et al. Pulmonary artery smooth muscle cell senescence is a pathogenic mechanism for pulmonary hypertension in chronic lung disease. Circ Res. 2011;109(5):543-553.

14. Jewell JL, Guan KL. Nutrient signaling to mTOR and cell growth. Trends Biochem Sci. 2013;38(5):233-242.

15. Lamming DW, Ye L, Sabatini DM, Baur JA. Rapalogs and mTOR inhibitors as anti-aging therapeutics. J Clin Invest. 2013;123(3):980-989.

16. Astle MV, et al. AKT induces senescence in human cells via mTORC1 and p53 in the absence of DNA damage: implications for targeting mTOR during malignancy. Oncogene. 2012;31(15):1949-1962.

17. Nogueira V, et al. Akt determines replicative senescence and oxidative or oncogenic premature senescence and sensitizes cells to oxidative apoptosis. Cancer Cell. 2008;14(6):458-470.

18. Harrison DE, et al. Rapamycin fed late in life extends lifespan in genetically heterogeneous mice. Nature. 2009;460(7253):392-395.

19. Wullschleger S, Loewith R, Hall MN. TOR signaling in growth and metabolism. Cell. 2006;124(3):471-484.

20. Foster KG, Fingar DC. Mammalian target of rapamycin (mTOR): conducting the cellular signaling symphony. J Biol Chem. 2010;285(19):14071-14077.

21. Laplante M, Sabatini DM. mTOR signaling in growth control and disease. Cell. 2012;149(2):274-293.

22. Walters HE, Deneka-Hannemann S, Cox LS. Reversal of phenotypes of cellular senescence by pan-mTOR inhibition. Aging (Albany NY). 2016;8(2):231-244.

23. Ferrand $M$, et al. Screening of a kinase library reveals novel pro-senescence kinases and their common NF- $\mathrm{B}$-dependent transcriptional program. Aging (Albany NY). 2015;7(11):986-1003.

24. Houssaini A, et al. Selective Tuberous Sclerosis Complex 1 Gene Deletion in Smooth Muscle Activates Mammalian Target of Rapamycin Signaling and Induces Pulmonary Hypertension. Am J Respir Cell Mol Biol. 2016;55(3):352-367.

25. Claxton S, Kostourou V, Jadeja S, Chambon P, Hodivala-Dilke K, Fruttiger M. Efficient, inducible Cre-recombinase activation in vascular endothelium. Genesis. 2008;46(2):74-80.

26. Perl AK, Wert SE, Nagy A, Lobe CG, Whitsett JA. Early restriction of peripheral and proximal cell lineages during formation of the lung. Proc Natl Acad Sci USA. 2002;99(16):10482-10487.

27. Dazert E, Hall MN. mTOR signaling in disease. Curr Opin Cell Biol. 2011;23(6):744-755.

28. Ilagan E, Manning BD. Emerging role of mTOR in the response to cancer therapeutics. Trends Cancer. 2016;2(5):241-251.

29. Gary-Bobo G, et al. Effects of HIV protease inhibitors on progression of monocrotaline- and hypoxia-induced pulmonary hypertension in rats. Circulation. 2010;122(19):1937-1947.

30. Abid S, et al. P21-dependent protective effects of a carbon monoxide-releasing molecule-3 in pulmonary hypertension. Arterioscler Thromb Vasc Biol. 2014;34(2):304-312.

31. Goncharov DA, et al. Mammalian target of rapamycin complex 2 (mTORC2) coordinates pulmonary artery smooth muscle cell metabolism, proliferation, and survival in pulmonary arterial hypertension. Circulation. 2014;129(8):864-874.

32. Savale L, et al. Shortened telomeres in circulating leukocytes of patients with chronic obstructive pulmonary disease. $A m J$ Respir Crit Care Med. 2009;179(7):566-571.

33. Tsuji T, Aoshiba K, Nagai A. Cigarette smoke induces senescence in alveolar epithelial cells. Am J Respir Cell Mol Biol. 2004;31(6):643-649.

34. Iglesias-Bartolome R, et al. mTOR inhibition prevents epithelial stem cell senescence and protects from radiation-induced mucositis. Cell Stem Cell. 2012;11(3):401-414.

35. Mitani A, Ito K, Vuppusetty C, Barnes PJ, Mercado N. Restoration of Corticosteroid Sensitivity in Chronic Obstructive Pulmonary Disease by Inhibition of Mammalian Target of Rapamycin. Am J Respir Crit Care Med. 2016;193(2):143-153.

36. To Y, et al. Targeting phosphoinositide-3-kinase-delta with theophylline reverses corticosteroid insensitivity in chronic obstructive pulmonary disease. Am J Respir Crit Care Med. 2010;182(7):897-904.

37. Yoshida T, et al. Rtp801, a suppressor of mTOR signaling, is an essential mediator of cigarette smoke-induced pulmonary injury and emphysema. Nat Med. 2010;16(7):767-773.

38. Acosta JC, et al. Chemokine signaling via the CXCR2 receptor reinforces senescence. Cell. 2008;133(6):1006-1018.

39. Freund A, Orjalo AV, Desprez PY, Campisi J. Inflammatory networks during cellular senescence: causes and consequences Trends Mol Med. 2010;16(5):238-246.

40. Laberge RM, et al. MTOR regulates the pro-tumorigenic senescence-associated secretory phenotype by promoting IL1A translation. Nat Cell Biol. 2015;17(8):1049-1061.

41. Liebow AA. Pulmonary emphysema with special reference to vascular changes. Am Rev Respir Dis. 1959;80(1, Part 2):67-93

42. Seimetz M, et al. Inducible NOS inhibition reverses tobacco-smoke-induced emphysema and pulmonary hypertension in mice. Cell. 2011;147(2):293-305.

43. Mühlfeld C, Hegermann J, Wrede C, Ochs M. A review of recent developments and applications of morphometry/stereology in lung research. Am J Physiol Lung Cell Mol Physiol. 2015;309(6):L526-L536.

44. Russell RC, Yuan HX, Guan KL. Autophagy regulation by nutrient signaling. Cell Res. 2014;24(1):42-57.

45. Saxton RA, Sabatini DM. mTOR Signaling in Growth, Metabolism, and Disease. Cell. 2017;169(2):361-371.

46. Yao H, et al. SIRT1 protects against emphysema via FOXO3-mediated reduction of premature senescence in mice. J Clin Invest. 2012;122(6):2032-2045

47. Lee IH, et al. A role for the NAD-dependent deacetylase Sirt1 in the regulation of autophagy. Proc Natl Acad Sci USA. 2008;105(9):3374-3379.

48. Kume S, et al. Calorie restriction enhances cell adaptation to hypoxia through Sirt1-dependent mitochondrial autophagy in mouse aged kidney. J Clin Invest. 2010;120(4):1043-1055.

49. Petrusca DN, et al. Smoking exposure induces human lung endothelial cell adaptation to apoptotic stress. Am J Respir Cell Mol Biol. 2014;50(3):513-525.

50. Stanley SE, et al. Telomerase mutations in smokers with severe emphysema. J Clin Invest. 2015;125(2):563-570.

51. Tsuji T, Aoshiba K, Nagai A. Alveolar cell senescence in patients with pulmonary emphysema. Am J Respir Crit Care Med. 2006;174(8):886-893. 
52. Jarrard JA, Linnoila RI, Lee H, Steinberg SM, Witschi H, Szabo E. MUC1 is a novel marker for the type II pneumocyte lineage during lung carcinogenesis. Cancer Res. 1998;58(23):5582-5589.

53. Vindrieux D, et al. Repression of PLA2R1 by c-MYC and HIF-2alpha promotes cancer growth. Oncotarget. 2014;5(4):1004-1013.

54. Mouraret N, et al. Role for telomerase in pulmonary hypertension. Circulation. 2015;131(8):742-755.

55. Drummond D, et al. Combined Effects of in Utero and Adolescent Tobacco Smoke Exposure on Lung Function in C57B1/6J Mice. Environ Health Perspect. 2017;125(3):392-399.

56. Knudsen L, Weibel ER, Gundersen HJ, Weinstein FV, Ochs M. Assessment of air space size characteristics by intercept (chord) measurement: an accurate and efficient stereological approach. J Appl Physiol. 2010;108(2):412-421. 